\title{
A consistent and stabilized continuous/discontinuous Galerkin method for fourth-order incompressible flow problems
}

\author{
A.G.B. Cruz ${ }^{\text {a,* }}$, E.G. Dutra do Carmo ${ }^{\text {b }}$, F.P. Duda ${ }^{a}$ \\ ${ }^{a}$ Mechanical Engineering Department-PEM/COPPE, Federal University of Rio de Janeiro, Ilha do Fundão, 21945-970, P.B. 68503, Rio de Janeiro, RJ, Brazil \\ ${ }^{\mathrm{b}}$ Nuclear Engineering Department-PEN/COPPE, Federal University of Rio de Janeiro, Ilha do Fundão, 21945-970, P.B. 68509, Rio de Janeiro, RJ, Brazil
}

\section{A R T I C L E I N F O}

\section{Article history:}

Received 27 March 2011

Accepted 3 May 2012

Available online 15 May 2012

\section{Keywords:}

Discontinuous Galerkin methods

Fourth-order problems

GLS stability

Second gradient

\begin{abstract}
A B S T R A C T
This paper presents a new consistent and stabilized finite-element formulation for fourthorder incompressible flow problems. The formulation is based on the $C^{0}$-interior penalty method, the Galerkin least-square (GLS) scheme, which assures that the formulation is weakly coercive for spaces that fail to satisfy the inf-sup condition, and considers discontinuous pressure interpolations. A stability analysis through a lemma establishes that the proposed formulation satisfies the inf-sup condition, thus confirming the robustness of the method. This lemma indicates that, at the element level, there exists an optimal or quasioptimal GLS stability parameter that depends on the polynomial degree used to interpolate the velocity and pressure fields, the geometry of the finite element, and the fluid viscosity term. Numerical experiments are carried out to illustrate the ability of the formulation to deal with arbitrary interpolations for velocity and pressure, and to stabilize large pressure gradients.
\end{abstract}

(c) 2012 Elsevier Inc. All rights reserved.

\section{Introduction}

Finite-element formulations for fourth-order differential operators require the introduction of $C^{1}$-finite element spaces, a feature that gives rise to difficulties in terms of computational implementation (see, for instance, [1-5]). This motivated Engel et al. [1] to develop the $C^{0}$-interior penalty methods (also known as continuous/discontinuous Galerkin methods) for fourth-order elliptic boundary value problems. These methods were subsequently investigated in many works, such as $[2,3,6-8]$.

On the other hand, standard finite-element formulations for incompressible flow problems require, due to their mixed nature (cf. [9]), the choice of interpolation spaces, for velocity and pressure, satisfying the inf-sup condition or Banach-Necas-Babuska condition [10] (cf. [9-12]). This forbids, for instance, the use of equal order approximations for velocity and pressure (cf. [12,13]). However, as it is well known (see, for instance, Gresho and Sani [12]), the violation of the inf-sup condition potentially introduces, among other pathologies, spurious oscillations of the pressure and locking of the velocity. An efficient approach to circumvent the difficulties imposed by the inf-sup condition consists in using the Galerkin least square (GLS) method, in which least square residuals of the governing equations are accounted for (cf. [14-19]). In particular, the use of the GLS method enables arbitrary choices of velocity and pressure interpolation spaces, which is generally desirable from the computational point of view.

\footnotetext{
* Corresponding author.

E-mail address: aguicruz@mecanica.ufrj.br (A.G.B. Cruz).
} 
In this paper, a consistent and stabilized finite-element formulation for fourth-order incompressible flow problems is proposed. Motivated by the aforementioned discussion, the formulation is based on the continuous/discontinuous Galerking and Least Square methods. Further, discontinuous pressure interpolations are adopted, which are, in comparison with continuous pressure interpolations, physically more appropriate for enforcing the incompressibility constraint at the element level $[11,12]$ and, in addition, enables a partial condensation of the degrees of freedom. A stability analysis using a lemma encompassing the inf-sup condition is also presented, showing that the proposed formulation is weakly coercive and, therefore, robust. This lemma also suggests that there exists an optimal or quasi optimal LS (least square) stabilization parameter, which is not necessarily the same for all elements of the mesh. Numerical experiments are provided to illustrate the efficacy of the formulation to deal with any combination of velocity-pressure elements as well as to stabilize high gradients of pressure.

A finite-element formulation for the problem considered here was advanced in Kim et al. [20]. As in the present paper, Kim et al. [20] based their formulation on the $C^{0}$-interior penalty method proposed in Engel et al. [1]. However, their treatment of the incompressibility does not take into account the inf-sup condition. Therefore, it is not clear whether they could prevent the well known pathologies associated with the violation of the inf-sup condition.

The remainder of this paper is organized as follows. In Section 2, we introduce the fourth-order model problem and the associated variational formulation. Section 3 presents the discontinuous Galerkin least square formulation for this model problem and the corresponding stability issue examined in Section 4. Numerical examples are presented in Section 5. Concluding and final remarks are provided in Section 6 .

\section{Model problem}

Let $\Omega \subset \mathbb{R}^{n}(n=2$ or 3$)$ be a domain with boundary $\Gamma$ Lipschitz continuous. Let $\Gamma_{\mathrm{D}}$ and $\Gamma_{\mathrm{N}}$ be such that

$$
\Gamma=\Gamma_{\mathrm{D}} \cup \Gamma_{\mathrm{N}} \text { and meas }\left(\Gamma_{\mathrm{D}} \cap \Gamma_{\mathrm{N}}\right)=0,
$$

where meas(.) denotes the positive Lebesgue measure. We consider the functions $\mathbf{f} \in L^{2}(\Omega)^{n}, \mathbf{g}_{0} \in H^{\frac{3}{2}}\left(\Gamma_{\mathrm{D}}\right)^{n} \cap C^{0}\left(\Gamma_{\mathrm{D}}\right)^{n}, \mathbf{g}_{1} \in H^{\frac{1}{2}}\left(\Gamma_{\mathrm{D}}\right)^{n}$, $\mathbf{h}_{0} \in L^{2}\left(\Gamma_{\mathrm{N}}\right)^{n}$ and $\mathbf{h}_{1} \in L^{2}\left(\Gamma_{\mathrm{N}}\right)^{n}$. Recall that the spaces $L^{2}(\Omega)^{n}=\left\{\mathbf{s}=\left(s_{1}, \ldots, s_{n}\right) ; s_{i} \in L^{2}(\Omega)\right\}, H^{\frac{v}{2}}\left(\Gamma_{\mathrm{D}}\right)^{n}=\left\{\mathbf{g}=\left(g_{1}, \ldots, g_{n}\right) ; g_{i} \in H^{\frac{v}{2}}\left(\Gamma_{\mathrm{D}}\right)\right.$ $(v=1,2,3 \ldots)\}, H^{v}(\Omega)$ and $H^{v}(\Omega)^{n}=\left\{\mathbf{w}=\left(w_{1}, \ldots, w_{n}\right) ; w_{i} \in H^{v}(\Omega) ; v=1,2,3 \ldots\right\}$ are Sobolev spaces as defined in [21].

We now introduce the fourth-order boundary value problem of concern here. It consists of finding the pair (u,p), belonging to $H^{2}(\Omega)^{n} \times L^{2}(\Omega)$ if $\eta>0$ or to $H^{1}(\Omega)^{n} \times L^{2}(\Omega)$ if $\eta=0$, that satisfies

$$
\begin{aligned}
& \eta \Delta(\Delta \mathbf{u})-\mu \Delta \mathbf{u}+\nabla p=\mathbf{f} \text { in } \Omega, \\
& \nabla \cdot \mathbf{u}=0 \text { in } \Omega, \\
& \mathbf{u}=\mathbf{g}_{0} \text { on } \Gamma_{\mathrm{D}}, \\
& \frac{\partial \mathbf{u}}{\partial n}=(\nabla \mathbf{u}) \mathbf{n}=\mathbf{g}_{1} \text { on } \Gamma_{\mathrm{D}} \text { if } \eta>0, \\
& \left(\mu \frac{\partial \mathbf{u}}{\partial n}-p \mathbf{n}\right)-\eta \frac{\partial(\Delta \mathbf{u})}{\partial n}=\mathbf{h}_{0} \text { on } \Gamma_{\mathrm{N}}, \\
& \eta \Delta \mathbf{u}=\mathbf{h}_{1} \text { on } \Gamma_{\mathrm{N}} \text { if } \eta>0,
\end{aligned}
$$

where $\Delta \mathbf{u}$ denotes the Laplacian of the vector $\mathbf{u}, \nabla \cdot \mathbf{u}$ denotes the divergence of $\mathbf{u}, \nabla \mathbf{u}$ denotes the gradient of $\mathbf{u}, \mathbf{n}$ denotes the outward normal unit vector defined almost everywhere on $\Gamma$, the dot "." denotes the usual inner product in $\mathbb{R}^{n}$, and $\eta \geqslant 0$ and $\mu>0$ are constants.

It should be noted that the Laplacian is considered in the weak sense as follows: we say that $\mathbf{u} \in L^{2}(\Omega)^{n}$ has weak Laplacian in $L^{2}(\Omega)^{n}$, if and only if, there exists $\Delta \mathbf{u} \in L^{2}(\Omega)^{n}$ such that

$$
\int_{\Omega} \Delta \mathbf{u} \cdot \eta d \Omega=\int_{\Omega} \mathbf{u} \cdot(\Delta \boldsymbol{\eta}) d \Omega \quad \forall \boldsymbol{\eta} \in C_{0}^{\infty}(\Omega)^{n}
$$

where $C_{0}^{\infty}(\Omega)^{n}$ is as defined in [21].

Notice that for $\eta=0$ the boundary value problem given by (2)-(7) is reduced to the classical Stokes flow problem. Otherwise, for $\eta>0$, it is similar to the governing equations for second-gradient and incompressible fluid, cf. [22,23]. In this case, the length scale $\ell:=\sqrt{\eta / \mu}$ is introduced, which opens a way to account for small scale effects [22] (see also [20]).

We now proceed the wide form of boundary value problem defined. Toward this end, we begin by noteworthy the appropriate functional spaces.

Since the conditions associated with $\mathbf{g}_{0}$ and $\mathbf{g}_{1}$ are essential, we define the solution set for the velocity $\mathbf{u}$ as follows:

$$
S_{u}=\left\{\begin{array}{c}
\left\{\mathbf{u} \in H^{2}(\Omega)^{n} ; \mathbf{u}=\mathbf{g}_{0} \text { and } \frac{\partial \mathbf{u}}{\partial n}=\mathbf{g}_{1} \text { on } \Gamma_{\mathrm{D}}\right\} \text { if } \eta>0 \\
\left\{\mathbf{u} \in H^{1}(\Omega)^{n} ; \mathbf{u}=\mathbf{g}_{0} \text { on } \Gamma_{\mathrm{D}}\right\} \text { if } \eta=0
\end{array}\right.
$$


and we define the corresponding space of the admissible variations as follows:

$$
V_{u}=\left\{\begin{array}{c}
\left\{\mathbf{v} \in H^{2}(\Omega)^{n} ; \mathbf{v}=0 \text { and } \frac{\partial \mathbf{v}}{\partial n}=\mathbf{0} \text { on } \Gamma_{\mathrm{D}}\right\} \text { if } \eta>0 \\
\left\{\mathbf{v} \in H^{1}(\Omega)^{n} ; \mathbf{v}=0 \text { on } \Gamma_{\mathrm{D}}\right\} \text { if } \eta=0
\end{array}\right.
$$

The solution set for the pressure $p$ and the corresponding space of the admissible variations are all given as follows:

$$
\begin{aligned}
S_{p} & =\left\{\begin{array}{c}
L^{2}(\Omega) \text { and meas }\left(\Gamma_{\mathrm{N}}\right)>0 \\
\left\{q \in L^{2}(\Omega) ; \int_{\Omega} q d \Omega=0\right\} \text { if } \operatorname{meas}\left(\Gamma_{\mathrm{N}}\right)=0
\end{array}\right. \\
V_{p} & =S_{p} .
\end{aligned}
$$

Defining the multilinear form

$$
A^{*}(\mathbf{u}, p, \mathbf{v}, q)=\int_{\Omega}(\eta \Delta \mathbf{w} \cdot \Delta \mathbf{v}+\mu \nabla \mathbf{u}: \nabla \mathbf{v}) d \Omega+\int_{\Omega} q(\nabla \cdot \mathbf{u}) d \Omega-\int_{\Omega} p(\nabla \cdot \mathbf{v}) d \Omega,
$$

and the linear functional

$$
l(\mathbf{v})=\left\{\begin{array}{c}
\int_{\Omega} \mathbf{f} \cdot \mathbf{v} d \Gamma+\int_{\Gamma_{\mathrm{N}}} \mathbf{h}_{0} \cdot \mathbf{v} d \Gamma+\int_{\Gamma_{\mathrm{N}}} \mathbf{h}_{1} \cdot \frac{\partial \mathbf{v}}{\partial n} d \Gamma \text { if } \eta>0 \\
\int_{\Omega} \mathbf{f} \cdot \mathbf{v} d \Gamma+\int_{\Gamma_{\mathrm{N}}} \mathbf{h}_{0} \cdot \mathbf{v} d \Gamma \text { if } \eta=0
\end{array}\right.
$$

the weak form of the boundary value problem defined by (2)-(7) is introduced as: find the pair $(\mathbf{u}, p) \in S_{u} \times S_{p}$ satisfying

$$
A^{*}(\mathbf{u}, p, \mathbf{v}, q)=l(\mathbf{v}) \quad \forall(\mathbf{v}, q) \in V_{u} \times V_{p} .
$$

\section{Finite element approximation}

\subsection{Discontinuous Galerkin formulation}

Finite-element methods for the boundary value problem given in the Section 2 require finite-element spaces with $C^{1}$-continuity [3-5,24]. Here, however, we adopt the interior penalty method proposed in [1], which uses the standard $C^{0}$-Lagrange finite-element and enforces the continuity of derivatives across element boundaries in a weak sense.

Let $M^{h}=\left\{\Omega_{1}, \ldots, \Omega_{n e}\right\}$ be a regular partition of $\Omega$ into ne non-degenerate finite elements $\Omega_{e}$. The partition $M^{h}$ is such that: $\Omega_{e}$ can be isoparametrically mapped into standard elements and $\Omega_{e} \cap \Omega_{e^{\prime}}=\emptyset$ if $e \neq e^{\prime} ; \Omega \cup \Gamma=\cup_{e=1}^{n e}\left(\Omega_{e} \cup \Gamma_{e}\right)$ and $\Gamma_{e e^{\prime}}=\Gamma_{e} \cap \Gamma_{e^{\prime}}$, where $\Gamma_{e}$ denotes the boundary of $\Omega_{e}$. We define the broken Sobolev spaces $H^{v, b}\left(M^{h}\right)$ and $H^{v, b, 1}\left(M^{h}\right)$ on the partition $M^{h}$ as follows:

$$
\begin{aligned}
& H^{v, b}\left(M^{h}\right)=\left\{\varphi: \Omega \mapsto \mathbb{R} ; \varphi_{e} \in H^{v}\left(\Omega_{e}\right) \forall \Omega_{e} \in M^{h}\right\}, \\
& H^{v, b, 1}\left(M^{h}\right)=\left\{\varphi \in H^{v, b} \cap H^{1}(\Omega)\right\},
\end{aligned}
$$

where $\varphi_{e}$ is the restriction of $\varphi$ to $\Omega_{e}$.

Let $k \geqslant 1, k \geqslant l \geqslant 0$ and $r \geqslant 0$ be integers and consider $\mathcal{P}^{r}\left(\Omega_{e}\right)$ the space of polynomials of degree less than or equal to $r$ restricted to the element $\Omega_{e}$. Introducing the finite-dimensional spaces

$$
\begin{aligned}
& H^{h, k}=\left\{\varphi \in H^{2, b, 1}\left(M^{h}\right) ; \varphi_{e} \in \mathcal{P}^{k}\left(\Omega_{e}\right)\right\}, \\
& H^{h, k, n}=\left\{\varphi=\left(\varphi_{i}\right)_{1 \leqslant i \leqslant n} ; \varphi_{i} \in H^{h, k}\right\}, \\
& L^{h, l}=\left\{\varphi \in H^{0, b}\left(M^{h}\right) ; \varphi_{e} \in \mathcal{P}^{l}\left(\Omega_{e}\right)\right\},
\end{aligned}
$$

we consider then the spaces $S_{u}^{h, k}, V_{u}^{h, k}, S_{p}^{h}$ and $V_{p}^{h}$ defined as follows:

$$
\begin{aligned}
& S_{u}^{h, k}=\left\{\mathbf{u}^{h} \in H^{h, k, n} ; \mathbf{u}^{h}=\mathbf{g}_{0}^{h} \text { on } \Gamma_{\mathrm{D}}\right\}, \\
& V_{u}^{h, k}=\left\{\mathbf{v}^{h} \in H^{h, k, n} ; \mathbf{v}^{h}=\mathbf{0} \text { on } \Gamma_{\mathrm{D}}\right\}, \\
& S_{p}^{h}=V_{p}^{h}=S_{p} \cap L^{h, l},
\end{aligned}
$$

where $\mathbf{g}_{0}^{h}$ is the usual interpolating of $\mathbf{g}_{0}$.

Note that we envisage functions which are continuous on the entire domain but discontinuous in first and higher-order derivatives on interior boundaries. Thus $S_{u}^{h, k}$ and $V_{u}^{h, k}$ are $C^{0}$-finite-element spaces. We assume specially that $S_{p}^{h}$ and $V_{p}^{h}$ are $C^{-1}$-finite-element spaces, that is, we interpolate the pressure discontinuously. 
The continuous/discontinuous Galerkin method for (15) is to find $\left(\mathbf{u}^{h}, p^{h}\right) \in S_{u}^{h, k} \times S_{p}^{h}$ such that

$$
A\left(\mathbf{u}^{h}, p^{h}, \mathbf{v}^{h}, q^{h}\right)=l\left(\mathbf{v}^{h}\right) \quad \forall\left(\mathbf{v}^{h}, q^{h}\right) \in V_{u}^{h, k} \times V_{p}^{h},
$$

where

$$
\begin{aligned}
& A\left(\mathbf{u}^{h}, p^{h}, \mathbf{v}^{h}, q^{h}\right)=\sum_{e=1}^{n e}\left\{\int_{\Omega_{e}} \mu \nabla \mathbf{u}_{e}^{h}: \nabla \mathbf{v}_{e}^{h} d \Omega+\int_{\Omega_{e}} \mu \ell^{2} \Delta \mathbf{u}_{e}^{h} \cdot \Delta \mathbf{v}_{e}^{h} d \Omega-\int_{\Omega_{e}} p_{e}^{h}\left(\nabla \cdot \mathbf{v}_{e}^{h}\right) d \Omega+\int_{\Omega_{e}} q_{e}^{h}\left(\nabla \cdot \mathbf{u}_{e}^{h}\right) d \Omega\right. \\
& +\sum_{e^{\prime}>e}\left(-\int_{\Gamma_{e e^{\prime}}} \frac{1}{2}\left(\mu \ell^{2} \Delta \mathbf{u}_{e}^{h}+\mu \ell^{2} \Delta \mathbf{u}_{e^{\prime}}^{h}\right) \cdot\left(\frac{\partial \mathbf{v}_{e}^{h}}{\partial n_{e}}+\frac{\partial \mathbf{v}_{e^{\prime}}^{h}}{\partial n_{e^{\prime}}}\right) d \Gamma+\int_{\Gamma_{e e^{\prime}}} \frac{1}{2}\left(\frac{\partial \mathbf{u}_{e}^{h}}{\partial n_{e}}+\frac{\partial \mathbf{u}_{e^{\prime}}^{h}}{\partial n_{e^{\prime}}}\right) \cdot\left(\mu \ell^{2} \Delta \mathbf{v}_{e}^{h}+\mu \ell^{2} \Delta \mathbf{v}_{e^{\prime}}^{h}\right) d \Gamma\right. \\
& \left.\left.+\int_{\Gamma_{e e^{\prime}}} \tau_{u}\left(\frac{\partial \mathbf{u}_{e}^{h}}{\partial n_{e}}+\frac{\partial \mathbf{u}_{e^{\prime}}^{h}}{\partial n_{e^{\prime}}}\right) \cdot\left(\frac{\partial \mathbf{v}_{e}^{h}}{\partial n_{e}}+\frac{\partial \mathbf{v}_{e^{\prime}}^{h}}{\partial n_{e^{\prime}}}\right) d \Gamma\right)-\int_{\Gamma_{e} \cap \Gamma_{\mathrm{D}}} \mu \ell^{2} \Delta \mathbf{u}_{e}^{h} \cdot \frac{\partial \mathbf{v}_{e}^{h}}{\partial n_{e}} d \Gamma+\int_{\Gamma_{e} \cap \Gamma_{\mathrm{D}}} \frac{\partial \mathbf{u}_{e}^{h}}{\partial n_{e}} \cdot \mu \ell^{2} \Delta \mathbf{v}_{e}^{h} d \Gamma+\int_{\Gamma_{e} \cap \Gamma_{\mathrm{D}}} \tau_{\mathrm{D}} \frac{\partial \mathbf{u}_{e}^{h}}{\partial n_{e}} \cdot \frac{\partial \mathbf{v}_{e}^{h}}{\partial n_{e}} d \Gamma\right\}, \\
& l\left(\mathbf{v}^{h}\right)=\sum_{e=1}^{n e}\left\{\int_{\Omega_{e}} \mathbf{f}_{e} \cdot \mathbf{v}_{e}^{h} d \Gamma+\int_{\Gamma_{e} \cap \Gamma_{\mathrm{N}}} \mathbf{h}_{e, 0} \cdot \mathbf{v}_{e}^{h} d \Gamma+\int_{\Gamma_{e} \cap \Gamma_{\mathrm{N}}} \mathbf{h}_{e, 1} \cdot \frac{\partial \mathbf{v}_{e}^{h}}{\partial n_{e}} d \Gamma+\int_{\Gamma_{e} \cap \Gamma_{\mathrm{D}}} \mathbf{g}_{e, 1} \cdot\left(\mu \ell^{2} \Delta \mathbf{v}_{e}^{h}\right) d \Gamma+\int_{\Gamma_{e} \cap \Gamma_{\mathrm{D}}} \tau_{\mathrm{D}} \mathbf{g}_{e, 1} \cdot \frac{\partial \mathbf{v}_{e}^{h}}{\partial n_{e}} d \Gamma\right\},
\end{aligned}
$$

and $\tau_{\mathrm{u}}$ and $\tau_{\mathrm{D}}$ are penalty parameters to be fixed.

From dimensional analysis we find that $\tau_{\mathrm{u}}=\tau_{\mathrm{D}}=C \mu \ell^{2} / h_{e e^{\prime}}$, where $h_{e e^{\prime}}$ is a characteristic element edge length and $C$ is a positive constant independent of the mesh parameters. This constant have to be chosen carefully to guarantee sufficiently accuracy and good convergence of the method [1,20,8,25]. For the purpose of this paper, the constant $C$ is at the moment determined by numerical experiments. It is noteworthy that a large penalty parameter adversely affects the accuracy of the $C^{0}$-interior penalty method [3].

On the other hand, the restrictions imposed by the inf-sup condition need to be verified for each particular choice of the finite-element spaces $S_{u}^{h, k}, S_{p}^{h}, V_{u}^{h, k}$ and $V_{p}^{h}$ to reach stabilized and convergent solutions. As is well known, quite few combinations of finite element pairs are able to fulfil the inf-sup condition within the Galerkin formulation (see, e.g., [12,13] for some stable mixed elements). However, these stable finite element methods for second order problems can not be stable for fourth-order problems (i.e, larger length scales $\ell$ ), which was circumvented in cf. [20] using an additional stabilization of the pressure.

\subsection{Consistency}

The Euler-Lagrange equations associated with the partition $M^{h}$ of the domain $\Omega$ are given by

$$
\begin{aligned}
& -\nabla p_{e}+\mu \Delta \mathbf{u}_{e}-\mu \ell^{2} \Delta \Delta \mathbf{u}_{e}+\mathbf{f}_{e}=0 \quad \text { in } \Omega_{e}, \\
& \nabla \cdot \mathbf{u}_{e}=0 \quad \text { in } \quad \Omega_{e}, \\
& \mathbf{u}_{e}=\mathbf{g}_{0} \quad \text { on } \Gamma_{e} \cap \Gamma_{\mathrm{D}} \quad \text { if meas }\left(\Gamma_{e} \cap \Gamma_{\mathrm{D}}\right)>0, \\
& \frac{\partial \mathbf{u}_{e}}{\partial n_{e}}=\left(\nabla \mathbf{u}_{e}\right) \mathbf{n}=\mathbf{g}_{1} \quad \text { on } \Gamma_{e} \cap \Gamma_{\mathrm{D}} \quad \text { if } \eta>0 \quad \text { end meas }\left(\Gamma_{e} \cap \Gamma_{\mathrm{D}}\right)>0, \\
& \left(\mu \frac{\partial \mathbf{u}_{e}}{\partial n_{e}}-p_{e} \mathbf{n}_{e}\right)-\frac{\partial\left(\mu \ell^{2} \Delta \mathbf{u}_{e}\right)}{\partial n_{e}}=\mathbf{h}_{e, 0} \quad \text { if meas }\left(\Gamma_{e} \cap \Gamma_{\mathrm{N}}\right)>0, \\
& \mu \ell^{2} \Delta \mathbf{u}_{e}=\mathbf{h}_{e, 1} \quad \text { on } \Gamma_{\mathrm{N}}, \quad \text { if } \operatorname{meas}\left(\Gamma_{e} \cap \Gamma_{\mathrm{N}}\right)>0, \\
& \frac{\partial \mathbf{u}_{e}}{\partial n_{e}}+\frac{\partial \mathbf{u}_{e^{\prime}}}{\partial n_{e^{\prime}}}=\mathbf{0} \quad \text { on } \quad \Gamma_{e e^{\prime}}, \\
& {\left[\left(\mu \frac{\partial \mathbf{u}_{e}}{\partial n_{e}}-p_{e} \mathbf{n}_{e}\right)-\frac{\partial\left(\mu \ell^{2} \Delta \mathbf{u}_{e}\right)}{\partial n_{e}}\right]+\left[\left(\mu \frac{\partial \mathbf{u}_{e^{\prime}}}{\partial n_{e^{\prime}}}-p_{e^{\prime}} \mathbf{n}_{e^{\prime}}\right)-\frac{\partial\left(\mu \ell^{2} \Delta \mathbf{u}_{e^{\prime}}\right)}{\partial n_{e^{\prime}}}\right]=\mathbf{0} \quad \text { on } \Gamma_{e e^{\prime}},} \\
& \mu \ell^{2} \Delta \mathbf{u}_{e}-\mu \ell^{2} \Delta \mathbf{u}_{e^{\prime}}=\mathbf{0} \quad \text { on } \Gamma_{e e^{\prime}},
\end{aligned}
$$

where $\mathbf{u}_{e}$ and $p_{e}$ are restrictions to $\Omega_{e}$.

After a successive integrating by parts we obtain 


$$
\begin{aligned}
0= & A\left(\mathbf{u}, p, \mathbf{v}^{h}, q^{h}\right)-l\left(\mathbf{v}^{h}\right)=\sum_{e=1}^{n e}\left\{\int_{\Omega_{e}}\left(\mu \Delta \mathbf{u}_{e}-\mu \ell^{2} \Delta \Delta \mathbf{u}_{e}-\nabla p_{e}+\mathbf{f}_{e}\right) \cdot \mathbf{v}_{e}^{h} d \Omega+\int_{\Omega_{e}} q_{e}^{h}\left(\nabla \cdot \mathbf{u}_{e}\right) d \Omega\right. \\
& +\sum_{e^{\prime}>e}\left(-\int_{\Gamma_{e e^{\prime}}} \frac{1}{2}\left(\frac{\partial \mathbf{u}_{e}}{\partial n_{e}}+\frac{\partial \mathbf{u}_{e^{\prime}}}{\partial n_{e^{\prime}}}\right) \cdot\left(\mu \ell^{2} \Delta \mathbf{v}_{e}^{h}+\mu \ell^{2} \Delta \mathbf{v}_{e^{\prime}}^{h}\right) d \Gamma+\int_{\Gamma_{e e^{\prime}}} \frac{1}{2}\left(\mu \ell^{2} \Delta \mathbf{u}_{e}-\mu \ell^{2} \Delta \mathbf{u}_{e^{\prime}}\right) \cdot\left(\frac{\partial \mathbf{v}_{e}^{h}}{\partial n_{e}}+\frac{\partial \mathbf{v}_{e^{\prime}}^{h}}{\partial n_{e^{\prime}}}\right) d \Gamma\right. \\
& \left.+\int_{\Gamma_{e^{\prime}}} \tau_{\mathrm{u}}\left(\frac{\partial \mathbf{u}_{e}}{\partial n_{e}}+\frac{\partial \mathbf{u}_{e^{\prime}}}{\partial n_{e^{\prime}}}\right) \cdot\left(\frac{\partial \mathbf{v}_{e}^{h}}{\partial n_{e}}+\frac{\partial \mathbf{v}_{e^{\prime}}^{h}}{\partial n_{e^{\prime}}}\right) d \Gamma\right)-\int_{\Gamma_{e} \cap \Gamma_{\mathrm{N}}} \frac{1}{2}\left(\left[\left(\mu \frac{\partial \mathbf{u}_{e}}{\partial n_{e}}-p_{e} \mathbf{n}_{e}\right)-\frac{\partial\left(\mu \ell^{2} \Delta \mathbf{u}_{e}\right)}{\partial n_{e}}\right]\right. \\
& \left.+\left[\left(\mu \frac{\partial \mathbf{u}_{e^{\prime}}}{\partial n_{e^{\prime}}}-p_{e^{\prime}} \mathbf{n}_{e^{\prime}}\right)-\frac{\partial\left(\mu \ell^{2} \Delta \mathbf{u}_{e^{\prime}}\right)}{\partial n_{e^{\prime}}}\right]\right) \cdot\left(\mathbf{v}_{e}^{h}+\mathbf{v}_{e^{\prime}}^{h}\right) d \Gamma-\int_{\Gamma_{e} \cap \Gamma_{\mathrm{N}}}\left(\left[\left(\mu \frac{\partial \mathbf{u}_{e}}{\partial n_{e}}-p_{e} \mathbf{n}_{e}\right)-\frac{\partial\left(\mu \ell^{2} \Delta \mathbf{u}_{e}\right)}{\partial n_{e}}\right]-\mathbf{h}_{e, 0}\right) \cdot \mathbf{v}_{e}^{h} d \Gamma \\
& \left.+\int_{\Gamma_{e} \cap \Gamma_{\mathrm{N}}}\left(\mu \ell^{2} \Delta \mathbf{u}_{e}-\mathbf{h}_{e, 1}\right) \cdot \frac{\partial \mathbf{v}_{e}^{h}}{\partial n_{e}} d \Gamma-\int_{\Gamma_{e} \cap \Gamma_{\mathrm{D}}}\left(\frac{\partial \mathbf{u}_{e}}{\partial n_{e}}-\mathbf{g}_{e, 1}\right) \cdot \mu \ell^{2} \Delta \mathbf{v}_{e}^{h} d \Gamma+\int_{\Gamma_{e} \cap \Gamma_{\mathrm{D}}} \tau_{D}\left(\frac{\partial \mathbf{u}_{e}}{\partial n_{e}}-\mathbf{g}_{e, 1}\right) \cdot \frac{\partial \mathbf{v}_{e}^{h}}{\partial n_{e}} d \Gamma\right\} .
\end{aligned}
$$

Since this equation holds for all $\left(\mathbf{v}^{h}, q^{h}\right) \in V_{u}^{h, k} \times V_{p}^{h}$, the continuous/discontinuous Galerkin formulation (16) is therefore consistent in the sense that

$$
A\left(\mathbf{u}, p, \mathbf{v}^{h}, q^{h}\right)=l\left(\mathbf{v}^{h}\right)
$$

\subsection{Galerkin least square stabilization}

To alleviate the need of satisfying the inf-sup condition, and to take advantage of using the discontinuous pressure interpolation as well, we adopted the GLS stabilization method. The technique consists in including additional terms in the Galerkin form, so as to enhance its stability. These terms are obtained by minimization of the square of the $L^{2}$-norm of the discrete residual within each element [26], multiplied by adequate regularization (or stabilization) parameters that will control the contribution of the least square part in the variational sentence (cf. [14-19], for instance).

The continuous/discontinuous Galerkin formulation (16) in the least square sense (hereafter referred to as CDGLS formulation) is to find $\left(\mathbf{u}^{h}, p^{h}\right) \in S_{u}^{h, k} \times S_{p}^{h}$ such that

$$
A^{h}\left(\mathbf{u}^{h}, p^{h}, \mathbf{v}^{h}, q^{h}\right)=l_{\text {tot }}\left(\mathbf{v}^{h}, q^{h}\right) \quad \forall\left(\mathbf{v}^{h}, q^{h}\right) \in V_{u}^{h, k} \times V_{p}^{h},
$$

with

$$
\begin{aligned}
& A^{h}\left(\mathbf{u}^{h}, p^{h}, \mathbf{v}^{h}, q^{h}\right)=A\left(\mathbf{u}^{h}, p^{h}, \mathbf{v}^{h}, q^{h}\right)+\sum_{e=1}^{n e} A_{L S}^{e}\left(\mathbf{u}^{h}, p^{h}, \mathbf{v}^{h}, q^{h}\right), \\
& l_{\text {tot }}\left(\mathbf{v}^{h}, q^{h}\right)=l\left(\mathbf{v}^{h}, q^{h}\right)+\sum_{e=1}^{n e} l_{L S}^{e}\left(\mathbf{v}^{h}, q^{h}\right),
\end{aligned}
$$

where the forth terms are the GLS terms

$$
\begin{aligned}
& A_{L S}^{e}(\mathbf{w}, q, \delta \mathbf{w}, \delta q)=\int_{\Omega_{e}} \delta_{\mathrm{GLS}}\left(h_{e}\right)\left(\sum_{i=1}^{n} R_{i}(\mathbf{w}, q) R_{i}(\delta \mathbf{w}, \delta q)\right) d \Omega, \\
& R_{i}(\mathbf{w}, q)=\frac{\partial p}{\partial x_{i}}-\mu \Delta w_{i}+\mu \ell^{2} \Delta \Delta w_{i}, \\
& l_{L S}^{e}\left(\mathbf{v}^{h}, q^{h}\right)=\int_{\Omega_{e}} \delta_{\mathrm{GLS}}\left(h_{e}\right)\left(\sum_{i=1}^{n} f_{i} R_{i}\left(\mathbf{v}^{h}, q^{h}\right)\right) d \Omega,
\end{aligned}
$$

with $(\mathbf{w}, q) \in H^{4, b}\left(M^{h}\right)^{n} \times H^{1, b}\left(M^{h}\right)$ and $(\delta \mathbf{w}, \delta q) \in H^{4, b}\left(M^{h}\right)^{n} \times H^{1, b}\left(M^{h}\right)$, and $\delta_{\mathrm{GLS}}\left(h_{e}\right)$ is the element stabilization parameter, which here will be defined by

$$
\delta_{\mathrm{GLS}}\left(h_{e}\right)=\frac{\alpha h_{e}^{2}}{\mu},
$$

where $\alpha$ is a dimensionless parameter to be fixed and $h_{e}$ is a characteristic length of the element usually given by $h_{e}=\left(\int_{\Omega_{e}} d \Omega\right)^{1 / n}$; cf., e.g., [17]. Note that the addition of these terms does not affect the consistency proof presented earlier. Note also that for triangular and tetrahedral elements up to order three, as well as for quadratic quadrilateral and hexahedral elements the fourth-order differential operator vanishes, which implies that any calculation involving derivatives of order greater than two is trivial for these elements. All these elements are widely used in practice.

As will be shown later, the CDGLS formulation (30) is stable, consistent and satisfy the inf-sup condition, thereby allowing and enables a wider choice of velocity and pressure spaces. Moreover, it enables the stabilization of large gradients of pressure, as clearly shown by the numerical results discussed in Section 5 . 
The dimensionless parameter $\alpha$ in (36) needs to be carefully determined to ensure sufficient accuracy and good convergency of GLS-stabilized formulations (cf. [27]). A methodology that allows to determine, at element level, optimal or quasioptimal parameters of the GLS-stabilized formulations for second-order incompressible problems has been investigated in Carmo et al. $[28,29]$. However, the extension of these results to fourth-order problems with incompressibility constraint is not trivial and require a careful analysis of stability. This issue will discussed in the next section.

\section{Stability analysis of the fourth-order incompressible problem}

We provide a stability analysis of the CDGLS-stabilized method (30) when applied to the fourth-order boundary value problem given in Section 2. The analysis suggests the existence of an appropriate stabilization parameter $\alpha$ that is dependent of the degree of the polynomial used to interpolate the velocity $\mathbf{u}$ and pressure $p$ fields, the geometry of the elements and the fluid viscosity term $\mu$. The result that enables to develop a methodology appropriate to determine this parameter is presented in the Lemma 1, which extends the results obtained in Carmo et al. [28,29] for second order incompressible problems.

We consider that the finite elements spaces have been constructed using meshes satisfying a regularity condition MC1, wherein the element distortion is controlled: there exist real constants $C_{1 \text {,distor }}>C_{0 \text {,distor }}>0$ such that

$$
C_{0, \text { distor }}<\frac{\rho_{e}}{h_{e}}<C_{1, \text { distor }} \forall \Omega_{e}
$$

where $\rho_{e}=\sup \left\{\operatorname{diam}(S), S\right.$ is a hypersphere contained in $\left.\Omega_{e} \cup \Gamma_{e}\right\}$ (cf. [10]). Owing to this control of mesh distortion, we find $c_{0}^{\prime}>0$ and $c_{1}^{\prime}>0$ to infer $c_{0}^{\prime} h_{e}<h_{e e^{\prime}}<c_{1}^{\prime} h_{e}, c_{0}^{\prime} h_{e^{\prime}}<h_{e e^{\prime}}<c_{1}^{\prime} h_{e}, c_{0}^{\prime} h_{e}<h_{e^{\prime}}<c_{1}^{\prime} h_{e}$ and $c_{0}^{\prime} h_{e^{\prime}}<h_{e}<c_{1}^{\prime} h_{e^{\prime}}$, where $h_{e e^{\prime}}=\min \left\{h_{e}, h_{e^{\prime}}\right\}$.

It follows from the well known theorem that says that all norms on a finite dimensional space are equivalent and under the regularity condition MC1 that the inverse inequalities hold: there are real constants $C_{\mathrm{Lap}, 1}>0, C_{\mathrm{Lap}, 2}>0$ and $C_{\text {bound, } 1}>0$ independent of the mesh parameter, depending only on the polynomial degree and the mesh distortion which is controlled by the constants $C_{0 \text {,distor }}$ and $C_{1 \text {,distor }}$ such that

$$
\begin{aligned}
& \int_{\Gamma_{e}} h_{e} \psi^{2} d \Omega \leqslant C_{\text {bound }, 1} \int_{\Omega_{e}} \psi^{2} d \Omega \quad \forall \psi \in \mathcal{P}^{k}\left(\Omega_{e}\right)(k \geqslant 1), \\
& \int_{\Omega_{e}} \ell^{2}|\Delta \varphi|^{2} d \Omega \leqslant \frac{\ell^{2}}{\left(h_{e}\right)^{2}} C_{\text {Lap }, 1} \int_{\Omega_{e}}|\nabla \varphi|^{2} d \Omega \quad \forall \varphi \in \mathcal{P}^{k}\left(\Omega_{e}\right)(k \geqslant 1), \\
& \int_{\Omega_{e}} \mu^{2} \ell^{4}|\Delta \Delta \varphi|^{2} d \Omega \leqslant \frac{\ell^{2}}{\left(h_{e}\right)^{2}} \frac{C_{\mathrm{Lap}, 2} \mu}{\left(h_{e}\right)^{2}} \int_{\Omega_{e}} \mu \ell^{2}|\Delta \varphi|^{2} d \Omega \quad \forall \varphi \in \mathcal{P}^{k}\left(\Omega_{e}\right)(k \geqslant 1) .
\end{aligned}
$$

We assume that for all $\mathbf{w}_{e} \in H^{1}\left(\Omega_{e}\right)^{n}$ and $q_{e} \in L^{2}\left(\Omega_{e}\right)$ the following decomposition at element level

$$
\mathbf{w}_{e}=\overline{\mathbf{w}}_{e}+\hat{\mathbf{w}}_{e^{\prime}} \text { and } q_{e}=\bar{q}_{e}+\hat{q}_{e},
$$

where

$$
\overline{\mathbf{w}}_{e}=\frac{\int_{\Omega_{e}} \mathbf{w}_{e} d \Omega}{\int_{\Omega_{e}} d \Omega} \quad \text { and } \quad \bar{q}_{e}=\frac{\int_{\Omega_{e}} q_{e} d \Omega}{\int_{\Omega_{e}} d \Omega} .
$$

Next, we consider the following Poincaré inequalities

$$
\begin{aligned}
& \forall \mathbf{w}_{e} \in H^{1}\left(\Omega_{e}\right)^{n} \quad\left\|\hat{\mathbf{w}}_{e}\right\|_{L^{2}\left(\Omega_{e}\right)^{n}} \leqslant C_{\text {Poinc }}^{e} h_{e}\left|\hat{\mathbf{w}}_{e}\right|_{H^{1}\left(\Omega_{e}\right)^{n}}, \\
& \forall q_{e} \in H^{1}\left(\Omega_{e}\right)^{n} \quad\left\|\hat{q}_{e}\right\|_{L^{2}\left(\Omega_{e}\right)^{n}} \leqslant C_{\text {Poinc }}^{e} h_{e}\left|\hat{q}_{e}\right|_{H^{1}\left(\Omega_{e}\right) .}
\end{aligned}
$$

where $|\cdot|_{H^{1}\left(\Omega_{e}\right)}$ and $|\cdot|_{H^{1}\left(\Omega_{e}\right)^{n}}$ denote seminorms of $H^{1}\left(\Omega_{e}\right)$ and $H^{1}\left(\Omega_{e}\right)^{n}$, respectively, and the positive real constant $C_{P o i n c}^{e}$ depending only on the usual aspect ratio which is controlled (MC1 condition) in finite elements meshes.

The result that follows is strongly inspired in [28,29] and represents an extension of the Lemma (4.38) of reference [10]. However, besides considering the two fundamental differences presented in [28,29], namely, (i) the parameter of stabilization now depends on $\Omega_{e}$ and, consequently, is not necessarily the same for all of the elements, (ii) the analysis is derived with a different and more appropriate norm to determine the stabilization parameter; we also extend these two differences to the fourth-order problems with internal incompressibility constraint.

To formalize this idea, for $0<\beta<1, \alpha_{e}>\alpha_{\min }>0 \forall \Omega_{e}$ and $k \geqslant 1$, we introduce the following norm on $V_{u}^{h, k} \times V_{p}^{h}$ :

$$
\begin{aligned}
& \left\|\left|\left(\mathbf{v}^{h}, q^{h}\right)\right|\right\|_{\beta, h, \Omega}^{2}=\sum_{e=1}^{n e}\left\{\frac{\beta}{(1+\beta)}\left|q^{h}\right|_{\alpha_{e}, h_{e}, \mu, H^{1}\left(\Omega_{e}\right)}^{2}+(1-\beta)\left(\left|\mathbf{v}^{h}\right|_{\mu, H^{1}\left(\Omega_{e}\right)^{n}}^{2}+\left|\mathbf{v}^{h}\right|_{\mu, \ell, \text { Lap }}^{2}+\sum_{e^{\prime}>e} \int_{\Gamma_{e e^{\prime}}} \tau_{v}\left|\left(\frac{\partial \mathbf{v}_{e}}{\partial n_{e}}+\frac{\partial \mathbf{v}_{e^{\prime}}}{\partial n_{e^{\prime}}}\right)\right|^{2} d \Gamma\right.\right. \\
& \left.\left.\quad+\sum_{i=1}^{n} \int_{\Omega_{e}} \frac{\alpha_{e}\left(h_{e}\right)^{2}}{\mu}\left|R_{i}\left(\mathbf{v}^{h}, q^{h}\right)\right|^{2} d \Omega\right)+\left\|\frac{\bar{q}_{e}^{h}}{\mu^{1 / 2}}\right\|_{L^{2}\left(\Omega_{e}\right)}^{2}\right\},
\end{aligned}
$$




$$
\begin{aligned}
& \left|\mathbf{v}^{h}\right|_{\mu, H^{1}\left(\Omega_{e}\right)^{n}}^{2}=\int_{\Omega_{e}}\left(\sum_{i=1}^{n} \mu\left|\nabla \mathbf{v}_{i}^{h}\right|^{2}\right) d \Omega, \\
& \left|\mathbf{v}^{h}\right|_{\mu, \ell, L a p}^{2}=\int_{\Omega_{e}}\left(\sum_{i=1}^{n} \mu \ell^{2}\left|\Delta \mathbf{v}_{i}^{h}\right|^{2}\right) d \Omega, \\
& \left|q^{h}\right|_{\alpha_{e}, h_{e}, \mu, H^{1}\left(\Omega_{e}\right)}^{2}=\int_{\Omega_{e}} \frac{\alpha_{e}\left(h_{e}\right)^{2}}{\mu}\left|\nabla q^{h}\right|^{2} d \Omega,
\end{aligned}
$$

which was extended here to the fourth-order problems.

Thus we summarize the discussion above into the following lemma.

Lemma 1. Under assumptions (MC1) and (38)-(40), if the parameter $\alpha_{e}$ is such that

$$
\begin{aligned}
& \alpha_{e}>\alpha_{\min }>0 \quad \forall \Omega_{e}, \\
& 2 \int_{\Omega_{e}} \frac{\alpha_{e}\left(h_{e}\right)^{2}}{\mu}\left(\mu \Delta u_{i}\right)^{2} d \Omega \leqslant \beta \int_{\Omega_{e}} \mu\left|\nabla u_{i}\right|^{2} d \Omega \quad \forall \Omega_{e} \quad \text { and } \quad \forall i, \\
& 2 \int_{\Omega_{e}} \frac{\alpha_{e}\left(h_{e}\right)^{2}}{\mu}\left(\mu \ell^{2} \Delta \Delta u_{i}\right)^{2} d \Omega \leqslant \beta \int_{\Omega_{e}} \mu \ell^{2}\left|\Delta u_{i}\right|^{2} d \Omega \quad \forall \Omega_{e} \quad \text { and } \quad \forall i,
\end{aligned}
$$

then there is $C_{\text {InfSup }}>0$ depending on $\left(\ell / h_{e}\right)$ such that

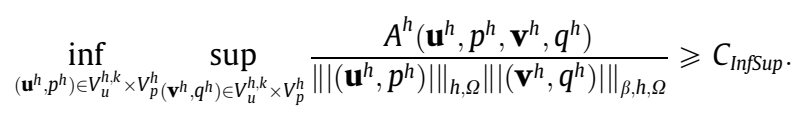

Proof. See Appendix A.

Remark 1. To proof Lemma 1 we assume discontinuous pressure interpolation. Formulations with discontinuous pressure interpolations may enforce the incompressibility condition strongly at the element level. However, one can prove, without additional difficulties, the stability for continuous pressure, cf. [29].

It can be observed from (52) and the proof of Lemma 1 that the CDGLS-stabilized formulation (30) is weak coercive with respect to the norm (45) for the discrete problem. Moreover, the results in Lemma 1 indicate a way to obtain a procedure to determine or bound optimal stability parameters.

\section{Numerical results}

The following numerical simulations illustrate the performance of the CDGLS formulation.

\subsection{Case 1: Plane Poiseuille flow}

We consider the steady pressure driven laminar flow between two stationary parallel plates that are separated by a fluid with viscosity $\mu$ and distance $d$. It is the most common type of flows observed in long, narrow channels (e.g, microfluidic

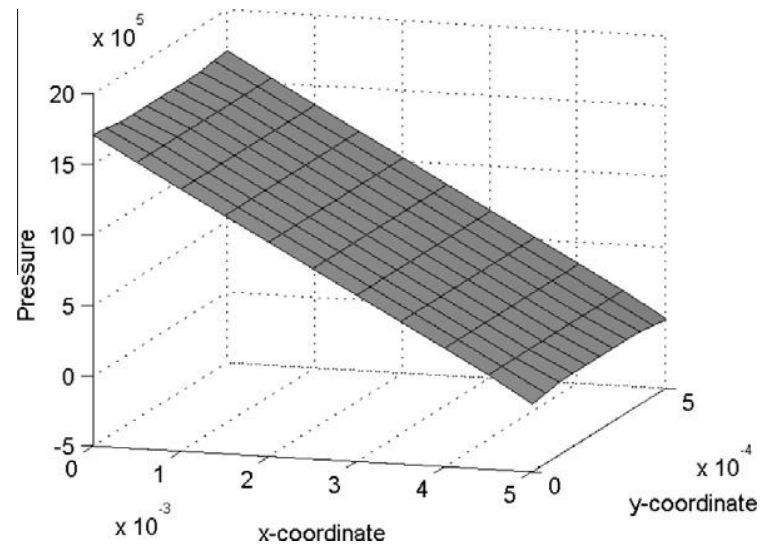

Fig. 1. Plane channel flow: stabilized pressure field by CDGLS scheme, $\alpha=1.5$, and Lagrangian $\mathbb{Q}_{2} / \mathbb{Q}_{1}$ finite element with discontinuous pressure. 


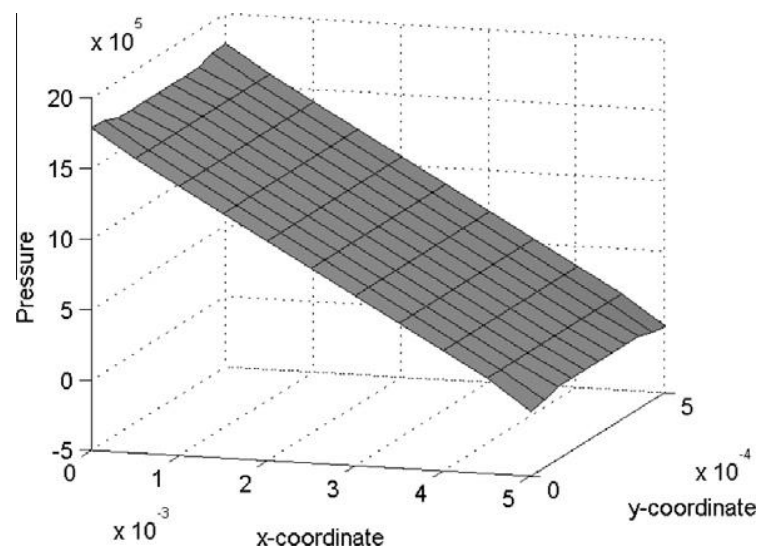

Fig. 2. Plane channel flow: stabilized pressure field by CDGLS scheme, $\alpha=0.05$, and Lagrangian $\mathbb{Q}_{2} / \mathbb{Q}_{1}$ finite element with discontinuous pressure.

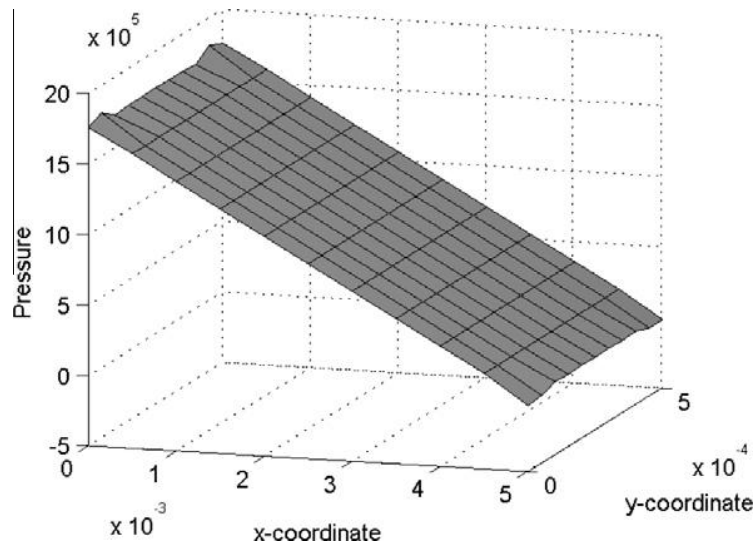

Fig. 3. Plane channel flow: stabilized pressure field by CDGLS scheme, $\alpha=0.005$, Lagrangian $\mathbb{Q}_{2} / \mathbb{Q}_{1}$ finite element with discontinuous pressure.

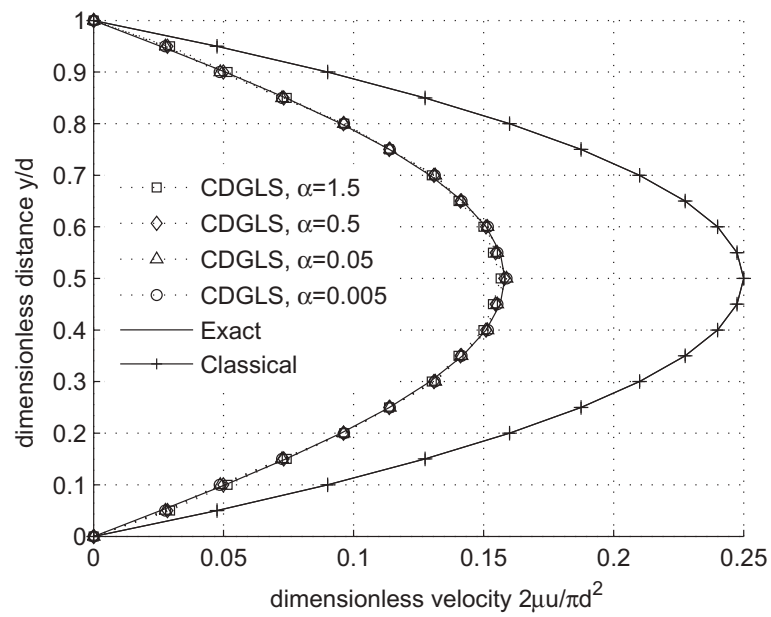

Fig. 4. Plane channel flow: exact and numerical velocity profiles across the centerline $x / d=0.5$ for different stabilization parameter $\alpha$ values. All numerical results were obtained with a mesh made up of 100 Lagrangian $\mathbb{Q}_{2} / \mathbb{Q}_{1}$ finite elements with discontinuous pressure.

devices) [30]. An analytical solution to this flow problem governed by the incompressible Eqs. (2) and (3) with no forcing term was developed by Fried and Gurtin in reference [22]. The pressure field is only known up to an arbitrary additive constant with gradient 


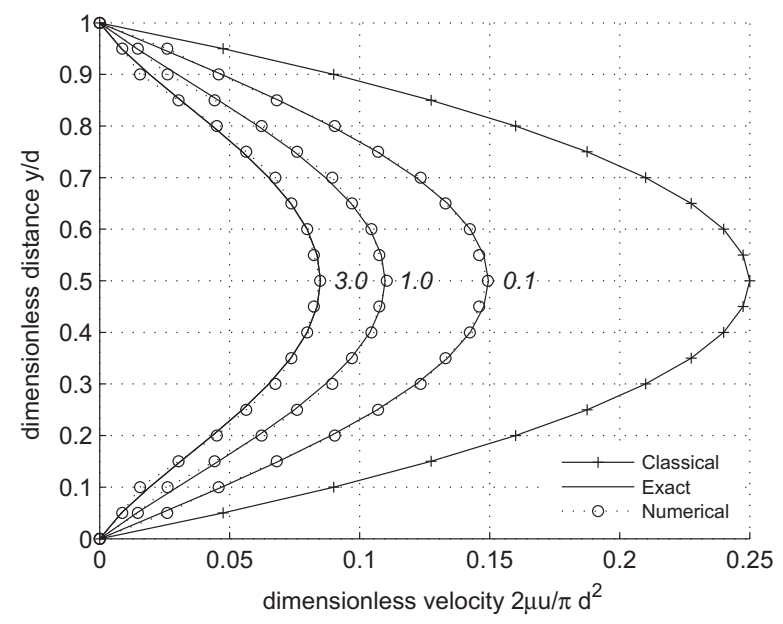

Fig. 5. Plane channel flow: exact and numerical profiles across $x / d=0.5$ for ratios $l / \ell$ equal to $0.1,1.0$ and 3.0 . All numerical results were obtained with a mesh made up of 100 Lagrangian $\mathbb{Q}_{2} / \mathbb{Q}_{1}$ elements with discontinuous pressure.

$$
\nabla p=-\pi \mathbf{e}_{x}
$$

and $\pi=$ constant, whereas the velocity solution $\mathbf{u}=u(y) \mathbf{e}_{x}$ is given by

$$
u(y)=\frac{\pi d^{2}}{2 \mu}\left\{\frac{y}{d}\left(1-\frac{y}{d}\right)-\frac{b_{l} \ell}{d \sinh (d / \ell)}\left(\sinh \frac{d}{\ell}-\sinh \frac{y}{\ell}-\sinh \frac{d-y}{\ell}\right)\right\},
$$

where

$$
b_{l}=\frac{(2 \ell / d)+(l / \ell)}{1+(l / \ell) \tanh (d / 2 \ell)}
$$

is a nonnegative dimensionless number, and $l>0$ is a adherence length scale; cf. [22]

As in reference [20], we use this exact solution which is essentially one-dimensional to construct a two-dimensional boundary value problem to verify the simulation results obtained with the CDGLS-stabilized scheme (30). We solved this flow problem in the rectangular channel domain $\Omega=[0,10 d] \times[0, d]$ with $d=0.5 \mathrm{~mm}$, prescribing the input boundary conditions $\mathbf{u}=u(y) \mathbf{e}_{x},(\nabla \mathbf{u}) \mathbf{n}=\mathbf{0}$ agreeing with exact velocity (54) on $\{x=0,0<y<d\}$ and output boundary conditions $u$ free, $v=0$, $(\nabla \mathbf{u}) \mathbf{n}=\mathbf{0}$ on $\{x=10 d, 0<y<d\}$. Fixed wall boundary conditions $\mathbf{u}=\mathbf{0},(\nabla \mathbf{u}) \mathbf{n}=\mathbf{g}_{1}$ were specified on both $\{y=0,0<x<10 d\}$, $\{y=d, 0<x<10 d\}$.

We begin by examining the numerical and exact solutions, and the sensitivity of the discrete solution for the stabilization parameter $\delta_{\mathrm{GLS}}$ trying several values of the parameter $\alpha$. For this, we set the material characteristic length $\ell$ equal to $d / 4$ and length scale $l=0$ are considered for the gradient theory. The spatial mesh is made up of 100 elements. The discrete solutions of the pressure field are shown in the Figs. 1-3 for $\mathbb{Q}_{2} / \mathbb{Q}_{1}$ finite element with discontinuous pressure. Fig. 4 shows the veloc-

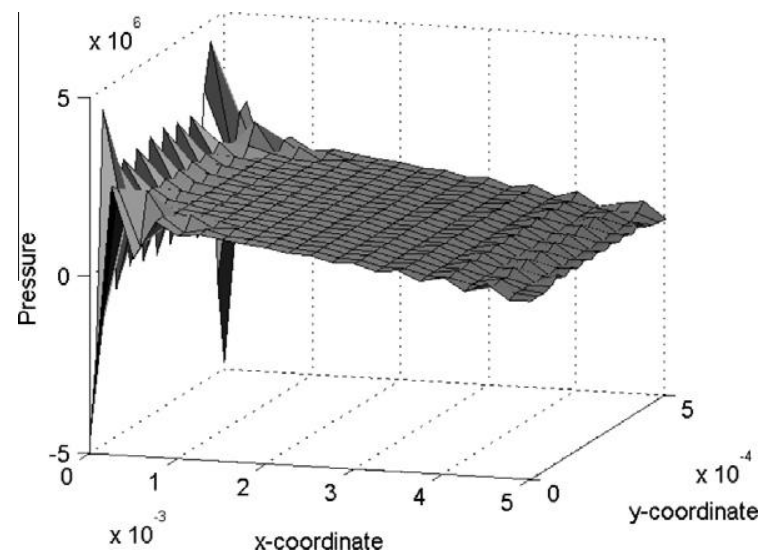

Fig. 6. Plane channel flow: oscillated pressure field by CDGLS scheme, $\alpha=0.000015$, and Lagrangian $\mathbb{Q}_{2} / \mathbb{Q}_{2}$ element with continuous pressure. 


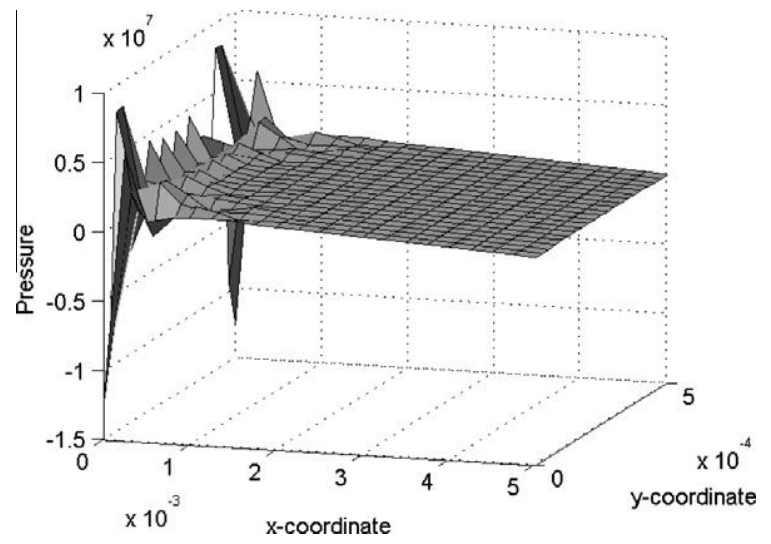

Fig. 7. Plane channel flow: oscillated pressure field by CDGLS scheme, $\alpha=0.000015$, and Lagrangian $\mathbb{Q}_{2} / \mathbb{Q}_{2}$ element with discontinuous pressure.

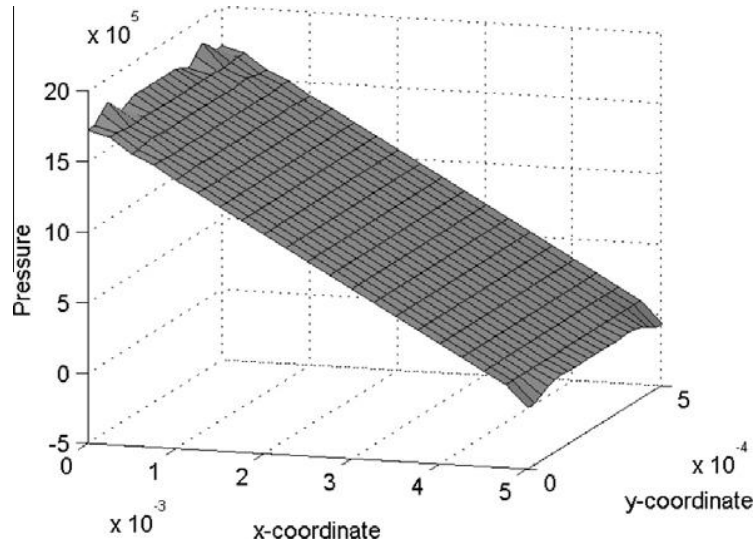

Fig. 8. Plane channel flow: stabilized pressure field by CDGLS scheme, $\alpha=0.05$, and Lagrangian $\mathbb{Q}_{2} / \mathbb{Q}_{2}$ element with discontinuous pressure.

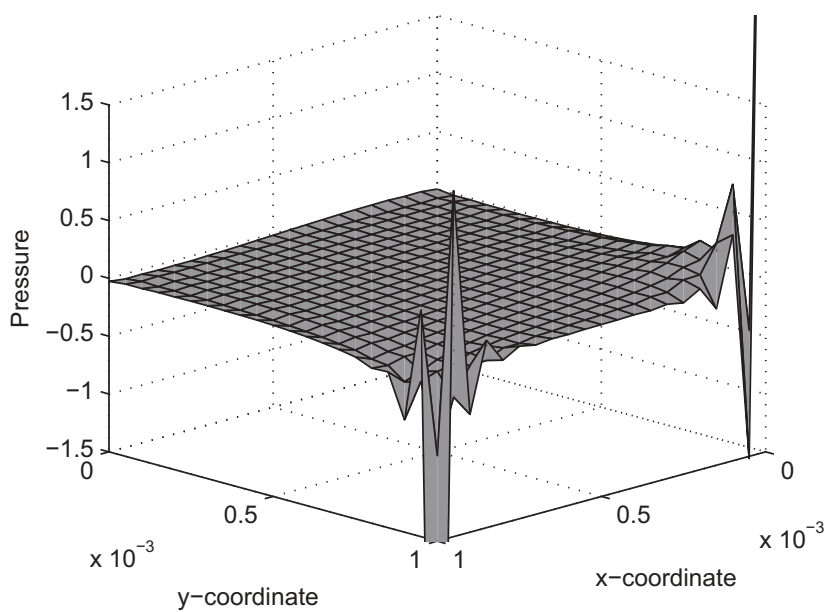

Fig. 9. Driven cavity flow: oscillated pressure field obtained with the Lagrangian $\mathbb{Q}_{2} / \mathbb{Q}_{1}$ element with continuous pressure; and stabilization parameter $\alpha=0.0005$ for the CDGLS method.

ity profiles across the center line $x / d=0.5$, together with exact and classical reference solution for this problem (cf. [22]), and are similar those obtained by Kim et al. [20]. Note that stability is reached for different $\alpha$ values. However, we may observe that for small values of $\alpha$ the pressure exhibits small perturbation in the corners of the domain. In the other extreme, if larger 


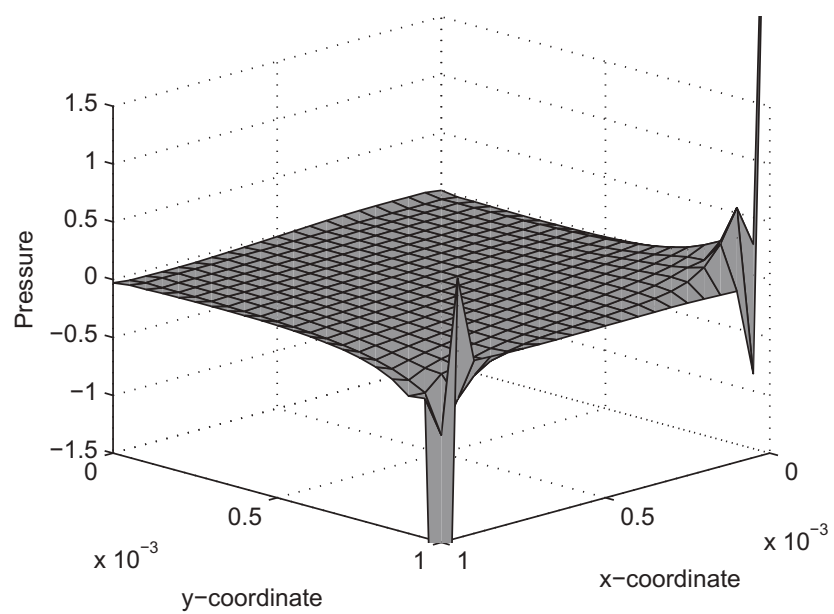

Fig. 10. Driven cavity flow: oscillated pressure field obtained with the Lagrangian $\mathbb{Q}_{2} / \mathbb{Q}_{1}$ element with discontinuous pressure; and stabilization parameter $\alpha=0.0005$ for the CDGLS method.

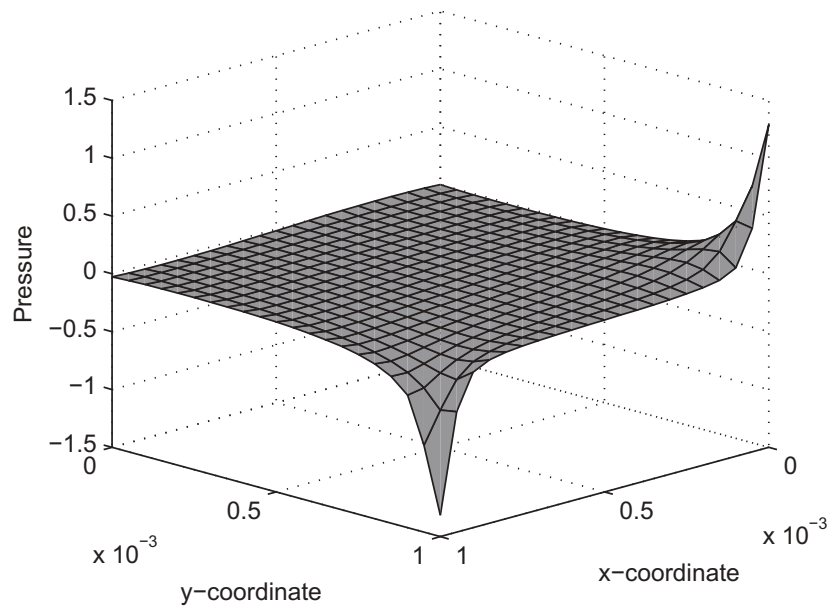

Fig. 11. Driven cavity flow: stabilized pressure field obtained with the Lagrangian $\mathbb{Q}_{2} / \mathbb{Q}_{1}$ element with discontinuous pressure; and stabilization parameter $\alpha=0.01$ for the CDGLS method.

values of $\alpha$ are used the velocity profiles present oscillations and do not reproduce the exact solution, while accompanied by reasonable pressure approximation.

Fig. 5 compares numerical and exact solutions across the channel center line $x / d=0.5$ for the case of generalized adherence conditions using three different ratios $l / \ell$ of adherence length to material length scale, compared with the classical reference solution for this problem. The results show a satisfactory match between the numerical and exact velocity fields, and are also similar those obtained in reference [20], It is expected that the velocity profiles at small length scales are smaller than those of conventional theory, as discussed in reference $[22,20]$.

Figs. 6 and 7 show the pressure field obtained with $\mathbb{Q}_{2} / \mathbb{Q}_{2}$ (biquadratic) element with continuous and discontinuous pressure interpolations, respectively, and they clearly depict the adverse effects of violating the inf-sup condition. Fig. 8 depicts that the pressure is stabilized, however, presenting slight perturbations in the corners of the computational domain.

\subsection{Case 2: Lid driven cavity flow problem}

In this second case, we solve the lid driven cavity flow problem in the square domain $\Omega=[0, d]^{2}$ at small length scale $(d=1 \mathrm{~mm})$ in which the velocity is set to zero and $(\nabla \mathbf{u}) \mathbf{n}=\mathbf{g}_{1}$ are enforced on all boundary except for the top lid $\{y=d, 0<x<d\}$, which moves with velocity $\mathbf{u}=U_{d} \mathbf{e}_{x}$ with speed $U_{d}$ constant; $(\nabla \mathbf{u}) \mathbf{n}=\mathbf{0}$. We also set the material length scale lequal to $1.5 d$, comparable to the geometric length scale $d$. and the Reynolds number was fixed igual to $2 \times 10^{-3}$.

We begin by considering the spatial mesh made up of $20 \times 20$ elements. Figs. 9 and 10 show oscillated pressure fields for the $\mathbb{Q}_{2} / \mathbb{Q}_{1}$ finite element by the CDGLS method with the pressure continuously and discontinuously interpolated, respec- 


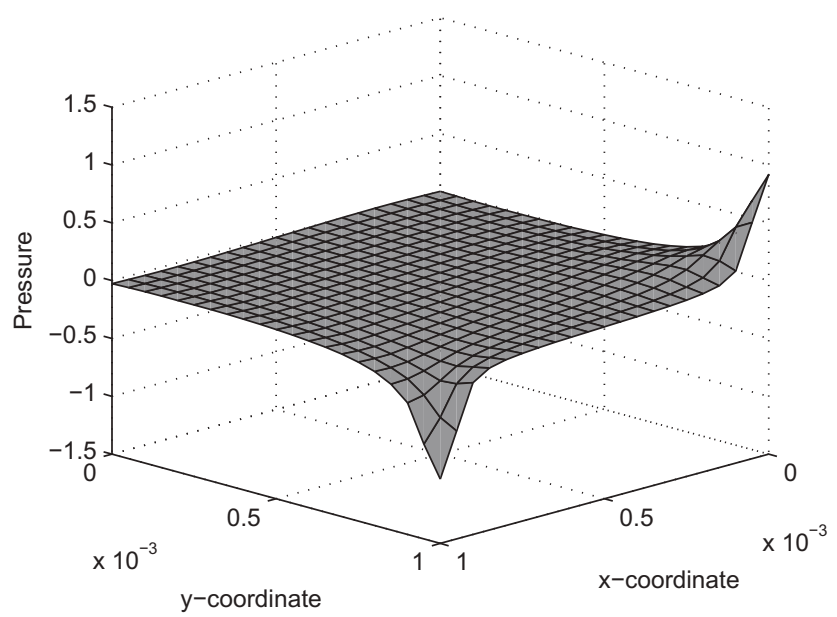

Fig. 12. Driven cavity flow: stabilized pressure field obtained with the Lagrangian $\mathbb{Q}_{2} / \mathbb{Q}_{1}$ element with discontinuous pressure; and stabilization parameter $\alpha=0.1$ for the CDGLS method.

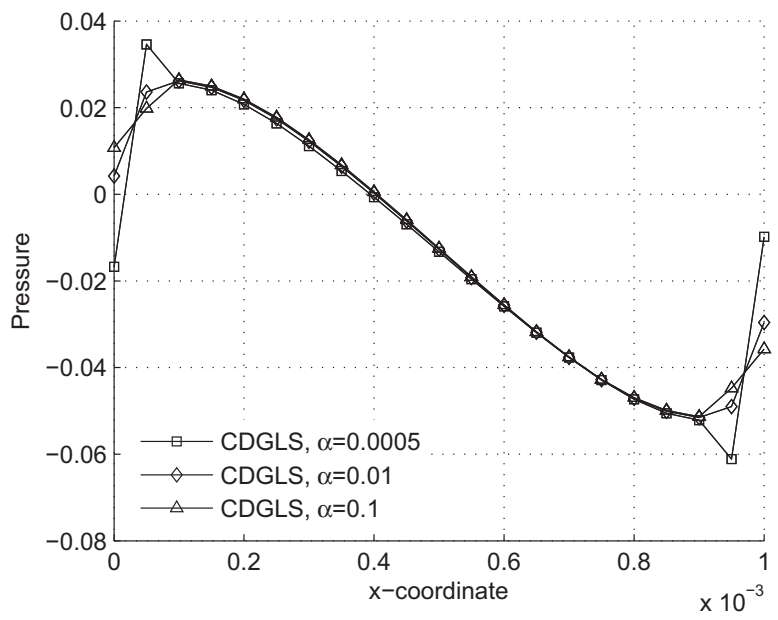

Fig. 13. Driven cavity flow: pressure distribution across the centerline $y=0.0005$; mesh made up of $20 \times 20$ elements.

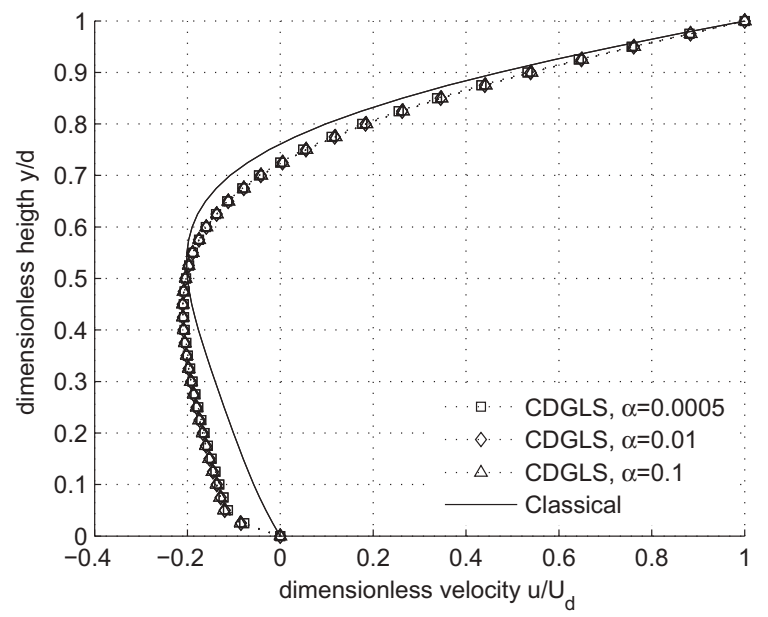

Fig. 14. Driven cavity flow: normalized velocity profiles across $y / d=0.5$; mesh made up of $20 \times 20$ elements. 


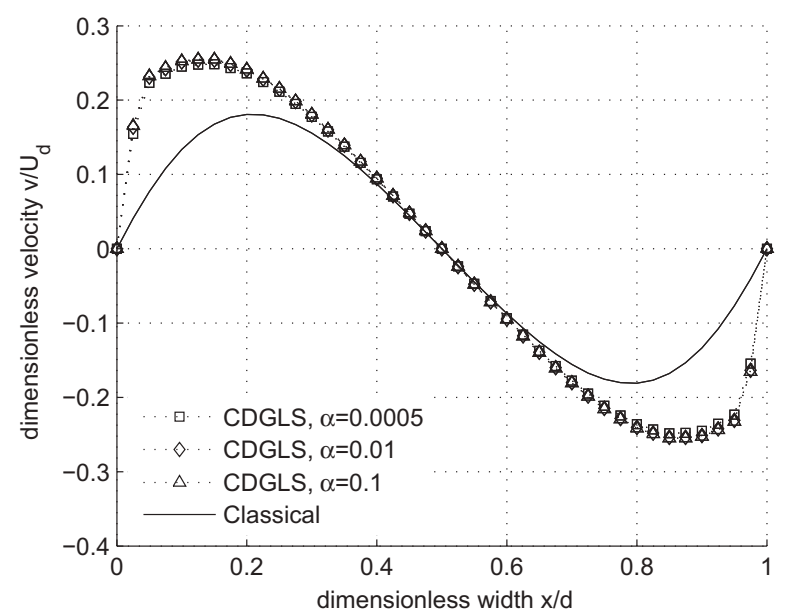

Fig. 15. Driven cavity flow: normalized velocity profiles across $x / d=0.5$; mesh made up of $20 \times 20$ elements.

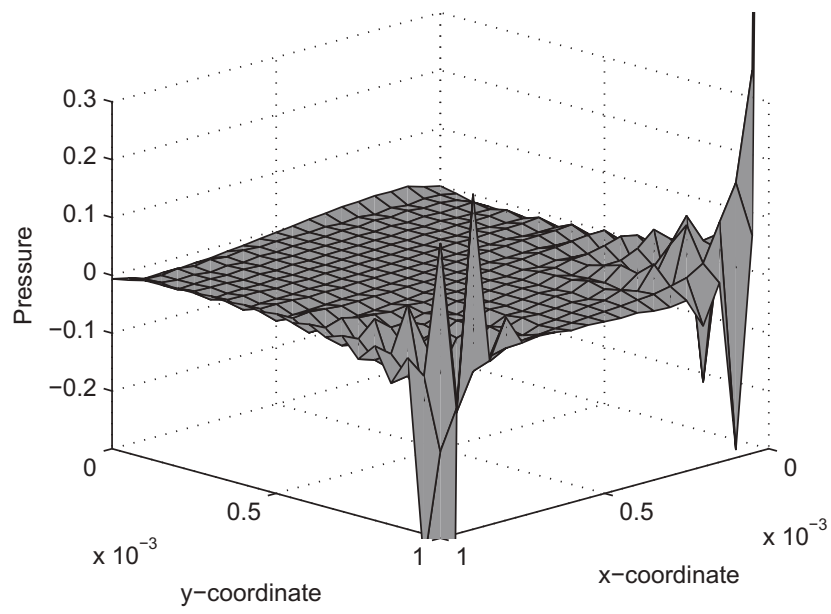

Fig. 16. Driven cavity flow: oscillated pressure field obtained with the Lagrangian $\mathbb{Q}_{2} / \mathbb{Q}_{2}$ element with continuous pressure, and stabilization parameter $\alpha=0.00025$ for the CDGLS method; mesh made up of $10 \times 10$ elements.

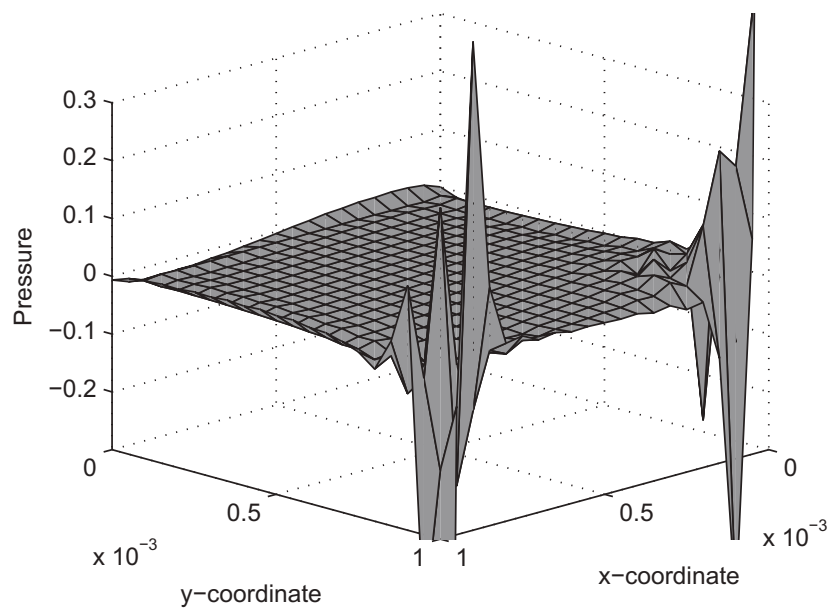

Fig. 17. Driven cavity flow: oscillated pressure field obtained with the Lagrangian $\mathbb{Q}_{2} / \mathbb{Q}_{2}$ element with discontinuous pressure, and stabilization parameter $\alpha=0.00025$ for the CDGLS method; mesh made up of $10 \times 10$ elements. 


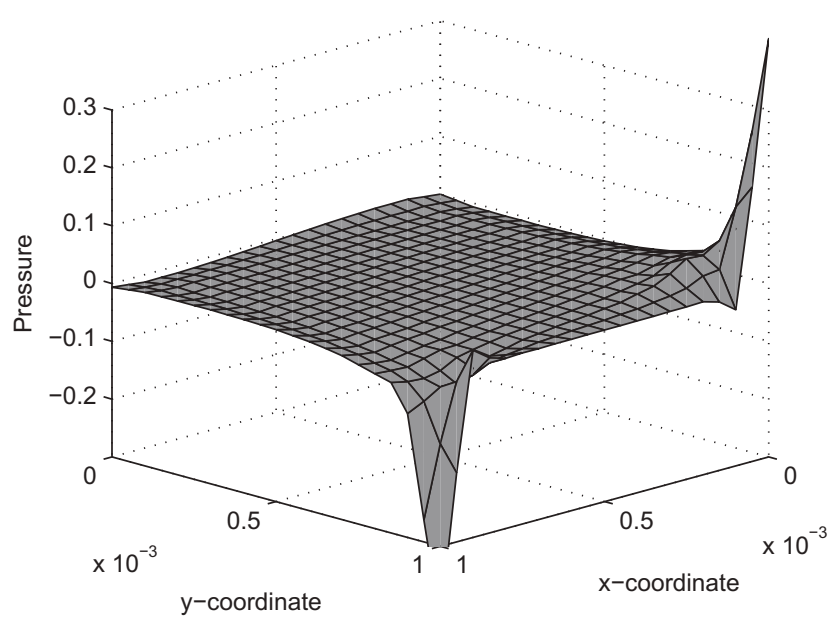

Fig. 18. Driven cavity flow: stabilized pressure field obtained with the Lagrangian $\mathbb{Q}_{2} / \mathbb{Q}_{2}$ element with discontinuous pressure, and stabilization parameter $\alpha=0.005$ for the CDGLS method; mesh made up of $10 \times 10$ elements.

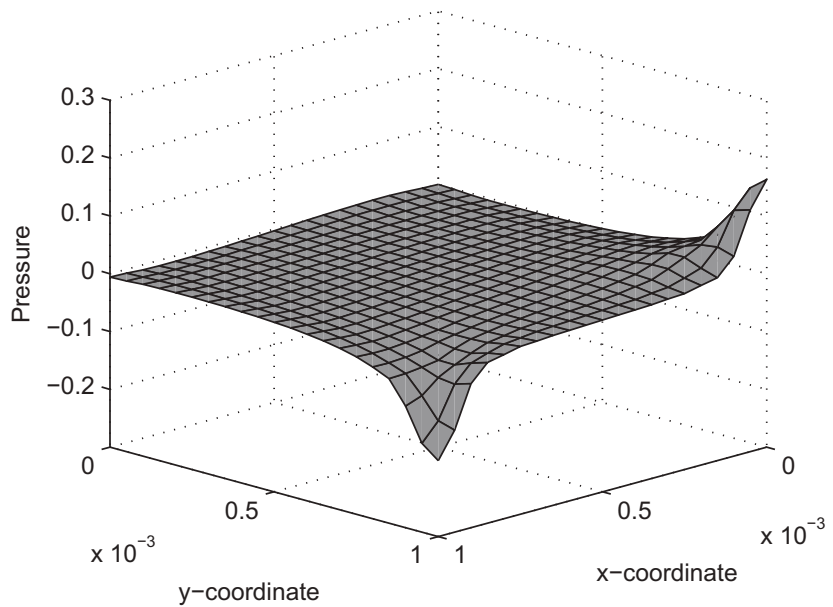

Fig. 19. Driven cavity flow: stabilized oscillated pressure field obtained with the Lagrangian $\mathbb{Q}_{2} / \mathbb{Q}_{2}$ element with discontinuous pressure, and stabilization parameter $\alpha=0.035$ for the CDGLS method; mesh made up of $10 \times 10$ elements.

tively. Figs. 11 and 12 show that the stability of the large pressure gradients can be reached gradually by using appropriate $\alpha$ values. It is important to observe that for a sufficiently small value of $\alpha$ the pressure is polluted by oscillations even for stable mixed interpolations. However, for large values of $\alpha$ there is the risk of over-stabilization by damping out discrete results of the pressure field changing the physics of the problem and the pressure on the corners of the domain might not be correctly captured (we recall that the pressure is singular at the top corners for this problem). Observations on this point agree quite well with those of the reference [27]. The pressure distribution along the horizontal line $y=0.0005$ is shown in Fig. 13, and accompanied by reasonable velocity approximations as shown by the velocity profiles across the centerlines $x / d=0.5$ and $y / d=0.5$ of Figs. 14 and 15.

We note that these results clearly indicate that the accuracy of the pressure field is sensitive to the stabilization parameter.

We conclude this section considering equal order approximations of velocity and pressure which are Galerkin unstable for second order problems; that is, do not satisfy the inf-sup condition. For this, we consider the spatial mesh made up of $10 \times 10$ elements. Pressure fields are shown in Figs. 16 and 17 for the biquadratic $\mathbb{Q}_{2} / \mathbb{Q}_{2}$ element with continuous and discontinuous pressure respectively. The pressure field obtained with $\mathbb{Q}_{2} / \mathbb{Q}_{2}$ element is polluted by spurious oscillations. These adverse effects are caused by violation of the inf-sup condition. The stability of the discrete pressure field can be reached gradually by using a suitable stabilization parameter $\alpha$ as shown in Figs. $18-20$. We note once again that for a large $\alpha$ the discrete solutions of the pressure field are very diffusive owing to over-stabilization of the large pressure gradients. Consequently, optimal or quasi optimal stabilization parameters have to be identified when using the CDGLS-stabilized method 


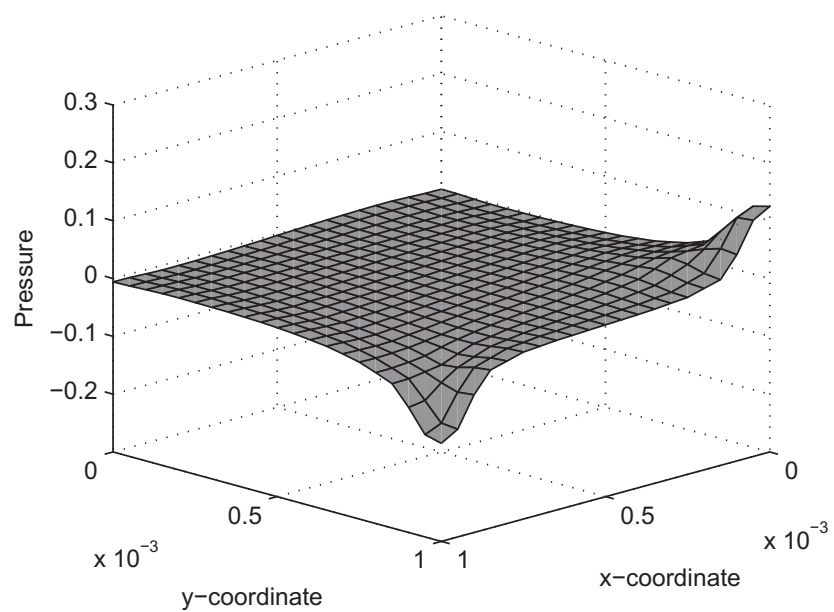

Fig. 20. Driven cavity flow: stabilized oscillated pressure field obtained with the Lagrangian $\mathbb{Q}_{2} / \mathbb{Q}_{2}$ element with discontinuous pressure, and stabilization parameter $\alpha=0.1$ for the CDGLS method; mesh made up of $10 \times 10$ elements.

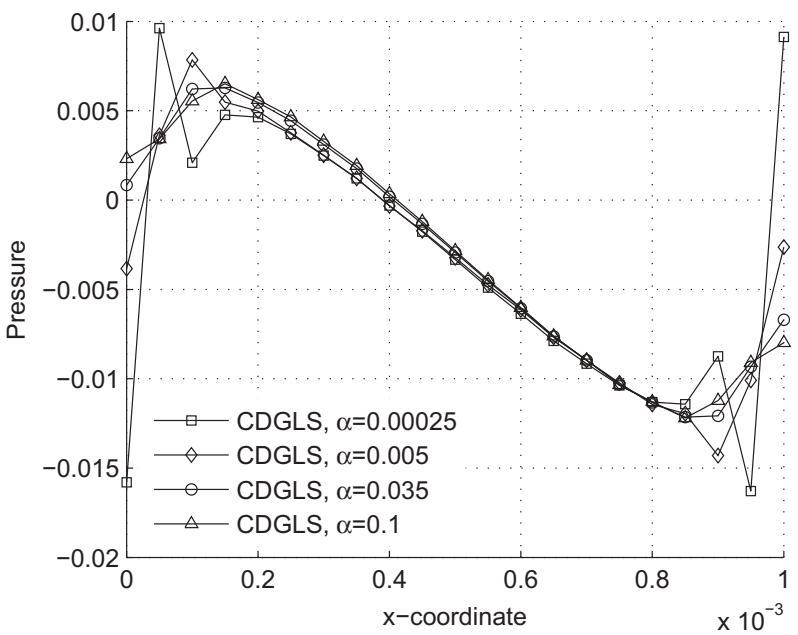

Fig. 21. Driven cavity flow: pressure distribution across the centerline $y=0.0005$; mesh made up of $10 \times 10$ elements.

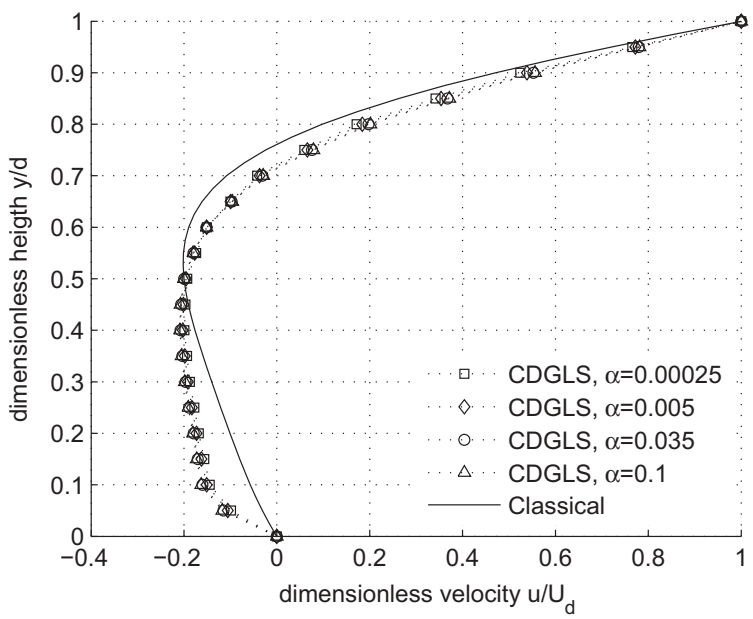

Fig. 22. Driven cavity flow: normalized velocity profile across $y / d=0.5$; mesh made up of $10 \times 10$ elements. 


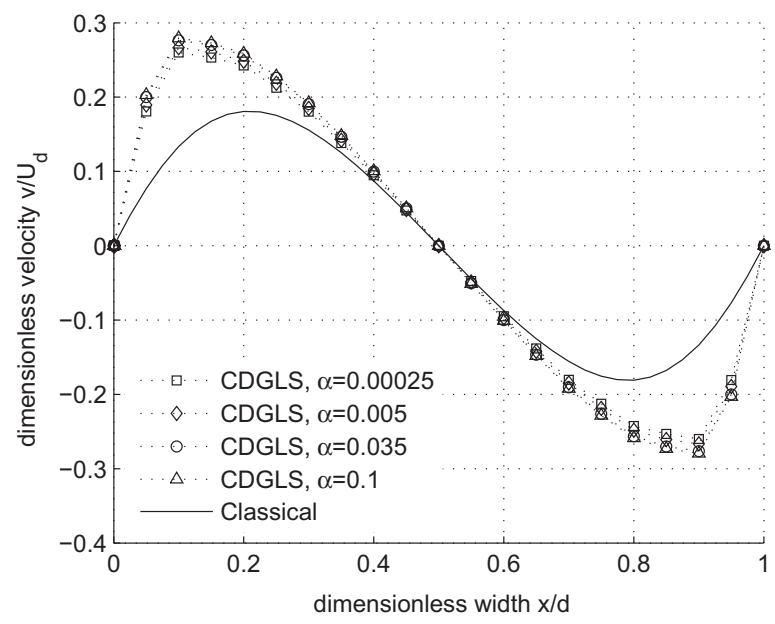

Fig. 23. Driven cavity flow: normalized velocity profile across $x / d=0.5$; mesh made up of $10 \times 10$ elements.

Fig. 21 shows the pressure distribution across the line $y / d=0.5$, without any significant loss for the velocity as shown by the velocity profiles across the cavity centerlines $x / d=0.5$ and $y / d=0.5$ of Figs. 22 and 23 respectively.

All these numerical results reinforce the importance of the GLS stabilization method in the proposed formulation. It can be observed that an optimum stabilization parameter needs to be obtained and that this parameter is not necessarily the same for all the elements in the mesh.

\section{Concluding remarks}

We have obtained an efficient and stabilized finite-element formulation for fourth-order incompressible flow problems. Our formulation is based on the $C^{0}$-interior penalty and the Galerkin least-square methods, and adopts discontinuous pressure interpolations. Furthermore, it is weakly coercive for discrete spaces that fail to satisfy the restrictions imposed by the inf-sup condition. Numerical results show that the proposed formulation can effectively stabilize large gradients of pressure, and also indicate that the choice of the discrete pressure and velocity spaces can be made freely. While this approach yields satisfactory results, the accuracy of the discrete results of the pressure is extremely sensitive to the stability parameter used in the GLS method. Therefore an optimum stabilization parameter has to be obtained to ensure accuracy and good convergency of the method.

A methodology to determine optimal or quasi-optimal stability parameters of GLS-stabilized finite element methods for second-order incompressible problems has been investigated in Carmo et al. [28,29]. However, the extension of these results to fourth-order problems with incompressibility constraint is not trivial. However the Lemma 1 indicates that there exists an optimal or quasi-optimal least square parameter that is not necessarily the same for all the elements in the mesh. It also asserts that this parameter depends on the degree of the polynomial used to interpolate the velocity and pressure fields, the geometry of the elements of the mesh and the fluid viscosity term.

From this analysis, we intend to extend the procedure proposed in $[28,29]$ to identify optimal stability parameters of GLS method applied to fourth-order problems with internal constraint. We also hope to be able to obtain a stability analysis with the inf-sup constant independent of the parameter $\left(\ell / h_{e}\right)$, together with a robust error estimate. It will be pursued in the near future.

\section{Acknowledgement}

The support from the Brazilian agency CAPES is gratefully acknowledged.

\section{Appendix A}

The following elementary inequality will be used in the proof of Lemma 1 below

$$
\pm a b \leqslant \frac{1}{2}\left(\gamma a^{2}+\frac{1}{\gamma} b^{2}\right) \quad \forall a, b \in \mathbb{R} \quad \text { and } \quad \forall \gamma \in \mathbb{R} \quad(\gamma>0) \text {. }
$$

Let $\lambda$ be defined as follows 


$$
\lambda=\lambda\left(\ell, M^{h}\right)=\max \left\{\left(\frac{\ell}{h_{e}}\right)^{2} ; e=1, \ldots, n e\right\} .
$$

Proof of Lemma 1. Let $\left(\mathbf{u}^{h}, p^{h}\right) \in V_{u}^{h, k} \times V_{p}^{h}$. The proof proceeds in three steps.

(1) A straightforward calculation yields

$$
\begin{aligned}
& A^{h}\left(\mathbf{u}^{h}, p^{h}, \mathbf{u}^{h}, p^{h}\right)=\sum_{e=1}^{n e}\left\{(1-\beta)\left(\left|\mathbf{u}^{h}\right|_{\mu, H^{1}\left(\Omega_{e}\right)^{n}}^{2}+\left|\mathbf{u}^{h}\right|_{\mu, \ell, L a p}^{2}+\sum_{e^{\prime}>e} \int_{\Gamma_{e^{\prime}}} \tau_{u}\left|\left(\frac{\partial \mathbf{u}_{e}}{\partial n_{e}}+\frac{\partial \mathbf{u}_{e^{\prime}}}{\partial n_{e^{\prime}}}\right)\right|^{2} d \Gamma+\sum_{i=1}^{n} \int_{\Omega_{e}} \frac{\alpha_{e}\left(h_{e}\right)^{2}}{\mu}\left|R_{i}\left(\mathbf{u}^{h}, p^{h}\right)\right|^{2} d \Omega\right)\right. \\
& \left.+\beta\left(\left|\mathbf{u}^{h}\right|_{\mu, H^{1}\left(\Omega_{e}\right)^{n}}^{2}+\left|\mathbf{u}^{h}\right|_{\mu,, \text { Lap }}^{2}+\sum_{e^{\prime}>e} \int_{\Gamma_{e e^{\prime}}} \tau_{u}\left|\left(\frac{\partial \mathbf{u}_{e}}{\partial n_{e}}+\frac{\partial \mathbf{u}_{e^{\prime}}}{\partial n_{e^{\prime}}}\right)\right|^{2} d \Gamma+\sum_{i=1}^{n} \int_{\Omega_{e}} \frac{\alpha_{e}\left(h_{e}\right)^{2}}{\mu}\left|R_{i}\left(\mathbf{u}^{h}, p^{h}\right)\right|^{2} d \Omega\right)\right\} .
\end{aligned}
$$

By using the inequality (A.1) with $\gamma=\beta$ in the following identity

$$
\frac{\beta}{(1+\beta)} \int_{\Omega_{e}} \frac{\alpha_{e}\left(h_{e}\right)^{2}}{\mu}\left(\frac{\partial p^{h}}{\partial x_{i}}\right)^{2}=\frac{\beta}{(1+\beta)} \int_{\Omega_{e}} \frac{\alpha_{e}\left(h_{e}\right)^{2}}{\mu}\left|R_{i}\left(\mathbf{u}^{h}, p^{h}\right)+\mu \Delta u_{i}^{h}-\mu \ell^{2} \Delta \Delta u_{i}^{h}\right|^{2} d \Omega,
$$

we have

$$
\begin{aligned}
& \frac{\beta}{(1+\beta)} \int_{\Omega_{e}} \frac{\alpha_{e}\left(h_{e}\right)^{2}}{\mu}\left(\frac{\partial p^{h}}{\partial x_{i}}\right)^{2} \leqslant \frac{\beta}{(1+\beta)}\left(\int_{\Omega_{e}} \frac{\alpha_{e}\left(h_{e}\right)^{2}}{\mu}(1+\beta)\left|R_{i}\left(\mathbf{u}^{h}, p^{h}\right)\right|^{2} d \Omega\right. \\
& \left.+\int_{\Omega_{e}} 2 \frac{\alpha_{e}\left(h_{e}\right)^{2}}{\mu}\left(1+\frac{1}{\beta}\right)\left(\left|\mu \Delta u_{i}^{h}\right|^{2}+\left|\mu \ell^{2} \Delta \Delta u_{i}^{h}\right|^{2}\right) d \Omega\right) .
\end{aligned}
$$

It follows from (46), (48), (50), (51) and (A.5) that

$$
\frac{\beta}{(1+\beta)}\left|p^{h}\right|_{\alpha_{e}, h_{e}, \mu, H^{1}\left(\Omega_{e}\right)}^{2} \leqslant \beta\left(\left|\mathbf{u}^{h}\right|_{\mu, H^{1}\left(\Omega_{e}\right)^{n}}^{2}+\left|\mathbf{u}^{h}\right|_{\mu, \ell, L a p}^{2}+\sum_{e^{\prime}>e} \int_{\Gamma_{e e^{\prime}}} \tau_{u}\left|\left(\frac{\partial \mathbf{u}_{e}}{\partial n_{e}}+\frac{\partial \mathbf{u}_{e^{\prime}}}{\partial n_{e^{\prime}}}\right)\right|^{2} d \Gamma+\int_{\Omega_{e}} \frac{\alpha_{e}\left(h_{e}\right)^{2}}{\mu}\left|R_{i}\left(\mathbf{u}^{h}, p^{h}\right)\right|^{2}\right) .
$$

Combining (45) and (A.3) with (A.6), we find

$$
A^{h}\left(\mathbf{u}^{h}, p^{h}, \mathbf{u}^{h}, p^{h}\right) \geqslant\left\|\mid\left(\mathbf{u}^{h}, p^{h}\right)\right\|_{\beta, h, \Omega}^{2}-\sum_{e=1}^{n e}\left(\left\|\frac{\bar{p}_{e}^{h}}{\mu^{1 / 2}}\right\|_{L^{2}\left(\Omega_{e}\right)}^{2}\right) .
$$

(2) $k>1\left(S_{p}^{h} \subset L^{2}(\Omega)\right.$ and $\left.V_{p}^{h} \subset L^{2}(\Omega)\right)$

From the result presented in Fortin [31] (cf. also [32]) and Lemma (4.19) given in reference [10], we have that for all $k>1$ there exists a positive real constant $C^{*, F o r t i n}>0$ such that, for all $p^{h} \in V_{p}^{h}$, there is $\mathbf{v}^{h} \in V_{u}^{h, k}$ satisfying

$$
\begin{aligned}
& \sum_{e=1}^{n e}-\left(\nabla \cdot \mathbf{v}_{e}^{h}, \bar{p}_{e}^{h}\right)_{L^{2}\left(\Omega_{e}\right)}=\sum_{e=1}^{n e}\left\|\frac{\bar{p}_{e}^{h}}{\mu^{1 / 2}}\right\|_{L^{2}\left(\Omega_{e}\right)}^{2}, \\
& \left\|\mathbf{v}^{h}\right\|_{H^{1}\left(\Omega_{e}\right)^{n}}^{2} \leqslant C^{*, F o r t i n}\left(\sum_{e=1}^{n e}\left\|\frac{\bar{p}_{e}^{h}}{\mu^{1 / 2}}\right\|_{L^{2}\left(\Omega_{e}\right)}^{2}\right)^{\frac{1}{2}} .
\end{aligned}
$$

Furthermore, using the inverse inequalities (38) and (39) together with the regularity condition MC1, and by inequality (A.1) with an appropriate value of $\gamma$ and the Fortin's result (A.9), after some algebraic manipulation, we have

$$
\begin{aligned}
& \sum_{e=1}^{n e}\left(\int_{\Omega_{e}} \mu \ell^{2}(\Delta \mathbf{v})^{2} d \Omega+\int_{\Omega_{e}} \mu|\nabla \mathbf{v}|^{2} d \Omega+\sum_{e^{\prime}>e} \int_{\Gamma_{e e^{\prime}}} \tau_{u}\left(\frac{\partial \mathbf{v}_{e}}{\partial n_{e}}+\frac{\partial \mathbf{v}_{e^{\prime}}}{\partial n_{e^{\prime}}}\right)^{2} d \Gamma\right) \\
& \leqslant C^{* *} \sum_{e=1}^{n e}\left(\left\|\mathbf{v}_{e}\right\|_{H^{1}\left(\Omega_{e}\right)^{n}}^{2}+\sum_{e^{\prime}>e}\left\|\mathbf{v}_{e^{\prime}}\right\|_{H^{1}\left(\Omega_{e}\right)^{n}}^{2}\right) \leqslant C^{* *}\left(N_{\text {face }}+1\right)\left(\sum_{e=1}^{n e}\left\|\mathbf{v}_{e}\right\|_{H^{1}\left(\Omega_{e}\right)^{n}}^{2}\right) \\
& \leqslant C^{* *}\left(N_{\text {face }}+1\right) C^{*, F o r t i n}\left(\sum_{e=1}^{n e}\left\|\frac{p_{e}}{\mu^{1 / 2}}\right\|_{L^{2}\left(\Omega_{e}\right)}^{2}\right)=\widetilde{C}^{2}\left(\sum_{e=1}^{n e}\left\|\frac{p_{e}}{\mu^{1 / 2}}\right\|_{L^{2}\left(\Omega_{e}\right)}^{2}\right)
\end{aligned}
$$

where $N_{\text {face }}$ is the number of faces of the element $\Omega_{e}$.

A straightforward calculation yields

$$
A^{h}\left(\mathbf{u}^{h}, p^{h}, \mathbf{v}^{h}, 0\right)=A^{\prime}\left(\mathbf{u}^{h}, \mathbf{v}^{h}\right)+\sum_{e=1}^{n e}\left(-\left(\nabla \cdot \mathbf{v}_{e}^{h}, \bar{p}_{e}^{h}+\hat{p}_{e}^{h}\right)_{L^{2}\left(\Omega_{e}\right)}+\sum_{i=1}^{n} \int_{\Omega_{e}} \frac{\alpha_{e}\left(h_{e}\right)^{2}}{\mu} R_{i}\left(\mathbf{u}^{h}, p^{h}\right)\left(\mu \Delta v_{i}^{h}\right) d \Omega_{e}\right),
$$




$$
\begin{aligned}
& A^{\prime}\left(\mathbf{u}^{h}, \mathbf{v}^{h}\right)=\sum_{e=1}^{n e}\left\{\sum_{1=1}^{n}\left(\int_{\Omega_{e}} \mu \nabla u_{e, i}^{h} \cdot \nabla v_{e, i}^{h} d \Omega_{e}+\int_{\Omega_{e}} \mu \ell^{2} \Delta u_{e, i}^{h} \Delta v_{e, i}^{h} d \Omega_{e}\right)\right. \\
& \quad+\sum_{e^{\prime}>e}\left(-\int_{\Gamma_{e^{\prime}}} \frac{1}{2}\left(\mu \ell^{2} \Delta \mathbf{u}_{e}^{h}+\mu \ell^{2} \Delta \mathbf{u}_{e^{\prime}}^{h}\right) \cdot\left(\frac{\partial \mathbf{v}_{e}}{\partial n_{e}}+\frac{\partial \mathbf{v}_{e^{\prime}}}{\partial n_{e^{\prime}}}\right) d \Gamma\right. \\
& \left.\left.+\int_{\Gamma_{e e^{\prime}}} \frac{1}{2}\left(\frac{\partial \mathbf{u}_{e}}{\partial n_{e}}+\frac{\partial \mathbf{u}_{e^{\prime}}}{\partial n_{e^{\prime}}}\right) \cdot\left(\mu \ell^{2} \Delta \mathbf{v}_{e}^{h}+\mu \ell^{2} \Delta \mathbf{v}_{e^{\prime}}^{h}\right) d \Gamma+\int_{\Gamma_{e e^{\prime}}} \tau_{u}\left(\frac{\partial \mathbf{u}_{e}}{\partial n_{e}}+\frac{\partial \mathbf{u}_{e^{\prime}}}{\partial n_{e^{\prime}}}\right) \cdot\left(\frac{\partial \mathbf{v}_{e}}{\partial n_{e}}+\frac{\partial \mathbf{v}_{e^{\prime}}}{\partial n_{e^{\prime}}}\right) d \Gamma\right)\right\} .
\end{aligned}
$$

Since

$$
\sum_{e=1}^{n e}\left\|\nabla \cdot \mathbf{v}_{e}^{h}\right\|_{L^{2}\left(\Omega_{e}\right)}^{2} \leqslant n \sum_{e=1}^{n e}\|\mathbf{v}\|_{H^{1}\left(\Omega_{e}\right)^{n}}^{2}
$$

by Cauchy-Schwartz inequality in $L^{2}\left(\Omega_{e}\right),(44),(49),\left(\right.$ A.8) and (A.9) and by Cauchy-Schwartz inequality in $\mathbb{R}^{\text {ne }}$, we have

$$
\begin{aligned}
& \sum_{e=1}^{n e}-\left(\nabla \cdot \mathbf{v}_{e}^{h}, \bar{p}_{e}^{h}+\hat{p}_{e}^{h}\right)_{L^{2}\left(\Omega_{e}\right)} \geqslant\left(\sum_{e=1}^{n e}\left\|\frac{\bar{p}_{e}^{h}}{\mu^{1 / 2}}\right\|_{L^{2}\left(\Omega_{e}\right)}^{2}\right)-C_{2}\left(\sum_{e=1}^{n e}\left\|\frac{\bar{p}_{e}^{h}}{\mu^{1 / 2}}\right\|_{L^{2}\left(\Omega_{e}\right)}^{2}\right)^{\frac{1}{2}}\left(\sum_{e=1}^{n e} \frac{\beta}{1+\beta}\left|p^{h}\right|_{\alpha_{e}, h_{e}, \mu_{,} H^{1}\left(\Omega_{e}\right)}^{2}\right)^{\frac{1}{2}}, \\
& C_{2}=C^{*, \text { Fortin }} \sup \left\{\left(\frac{n \mu(1+\beta)}{\beta \alpha_{e}}\right)^{\frac{1}{2}} C_{\text {Poinc }}^{e} ; e=1, \ldots, n e\right\} .
\end{aligned}
$$

Similarly, by Cauchy-Schwartz inequality in $L^{2}\left(\Omega_{e}\right),(50),\left(\right.$ A.9) and by Cauchy-Schwartz inequality in $\mathbb{R}^{\text {ne }}$, we have

$$
\begin{aligned}
\sum_{e=1}^{n e} \sum_{i=1}^{n} \int_{\Omega_{e}} \frac{\alpha_{e}\left(h_{e}\right)^{2}}{\mu} R_{i}\left(\mathbf{u}^{h}, p^{h}\right)\left(\mu \Delta v_{i}^{h}\right) \geqslant & -\left(\mu \frac{\beta}{1-\beta}\right)^{\frac{1}{2}} C^{* \text { Fortin }}\left(\left.\sum_{e=1}^{n e}|| \frac{\bar{p}_{e}^{h}}{\mu^{1 / 2}}\right|_{L^{2}\left(\Omega_{e}\right)} ^{2}\right)^{\frac{1}{2}} \\
& \times\left(\sum_{e=1}^{n e}(1-\beta) \sum_{i=1}^{n} \int_{\Omega_{e}} \frac{\alpha_{e}\left(h_{e}\right)^{2}}{\mu}\left|R_{i}\left(\mathbf{u}^{h}, p^{h}\right)\right|^{2}\right)^{\frac{1}{2}} .
\end{aligned}
$$

Next, by using the Cauchy-Schwartz inequality in $L^{2}\left(\Omega_{e}\right)$, the inequalities relations defined in (MC1), (38) and by CauchySchwartz inequality in $\mathbb{R}^{n e}$, we can find a constant $C_{1}^{c}>0$ such that

$$
\begin{aligned}
& A^{\prime}\left(\mathbf{u}^{h}, \mathbf{v}^{h}\right) \geqslant-C_{1}^{c}\left\{\sum_{e=1}^{n e}\left(\sum_{1=1}^{n} \int_{\Omega_{e}}\left(\mu\left(\nabla u_{e, i}^{h}\right)^{2} d \Omega_{e}+\mu \ell^{2}\left(\Delta u_{e, i}^{h}\right)^{2}\right) d \Omega_{e}+\sum_{e^{\prime}>e} \int_{\Gamma_{e e^{\prime}}} \tau_{u}\left(\frac{\partial \mathbf{u}_{e}}{\partial n_{e}}+\frac{\partial \mathbf{u}_{e^{\prime}}}{\partial n_{e^{\prime}}}\right)^{2} d \Gamma\right)\right\}^{\frac{1}{2}} \\
& \quad \times\left\{\sum_{e=1}^{n e}\left(\sum_{1=1}^{n} \int_{\Omega_{e}}\left(\mu\left(\nabla v_{e, i}^{h}\right)^{2}+\mu \ell^{2}\left(\Delta v_{e, i}^{h}\right)^{2}\right) d \Omega_{e}+\sum_{e^{\prime}>e} \int_{\Gamma_{e e^{\prime}}} \tau_{u}\left(\frac{\partial \mathbf{v}_{e}}{\partial n_{e}}+\frac{\partial \mathbf{v}_{e^{\prime}}}{\partial n_{e^{\prime}}}\right)^{2} d \Gamma\right)\right\}^{\frac{1}{2}}
\end{aligned}
$$

It follows from the result (A.10) that

$$
\begin{aligned}
A^{\prime}\left(\mathbf{u}^{h}, \mathbf{v}^{h}\right) \geqslant & -C_{1}^{c} \widetilde{C}\left((1-\beta) \sum_{e=1}^{n e}\left(\sum_{1=1}^{n} \int_{\Omega_{e}}\left(\mu\left(\nabla u_{e, i}^{h}\right)^{2}+\mu \ell^{2}\left(\Delta u_{e, i}^{h}\right)^{2}\right) d \Omega_{e}+\sum_{e^{\prime}>e} \int_{\Gamma_{e e^{\prime}}} \tau_{u}\left(\frac{\partial \mathbf{u}_{e}}{\partial n_{e}}+\frac{\partial \mathbf{u}_{e^{\prime}}}{\partial n_{e^{\prime}}}\right)^{2} d \Gamma\right)\right)^{\frac{1}{2}} \\
& \times\left(\frac{1}{(1-\beta)} \sum_{e=1}^{n e}\left\|\frac{\bar{p}_{e}^{h}}{\mu^{1 / 2}}\right\|_{L^{2}\left(\Omega_{e}\right)}^{2}\right)^{\frac{1}{2}} .
\end{aligned}
$$

From (A.11), (A.14)-(A.18) and by Cauchy-Schwartz inequality in $\mathbb{R}^{3}$, we infer that

$$
\begin{aligned}
& A^{h}\left(\mathbf{u}^{h}, p^{h}, \mathbf{v}^{h}, 0\right) \geqslant\left(\sum_{e=1}^{n e}\left\|\frac{\bar{p}_{e}^{h}}{\mu^{1 / 2}}\right\|_{L^{2}\left(\Omega_{e}\right)}^{2}\right)-C_{3}\left(\sum_{e=1}^{n e}\left\|\frac{\bar{p}_{e}^{h}}{\mu^{1 / 2}}\right\|_{L^{2}\left(\Omega_{e}\right)}^{2}\right)^{\frac{1}{2}} \\
& \times\left(\sum _ { e = 1 } ^ { n e } ( 1 - \beta ) \left(\left|\mathbf{u}^{h}\right|_{\mu, H^{1}\left(\Omega_{e}\right)}^{2}+\left|\mathbf{u}^{h}\right|_{\mu, \ell, \text { Lap }}^{2}+\sum_{i=1}^{n} \int_{\Omega_{e}} \frac{\alpha_{e}\left(h_{e}\right)^{2}}{\mu}\left|R_{i}\left(\mathbf{u}^{h}, p^{h}\right)\right|^{2} d \Omega\right.\right. \\
&\left.\left.+\sum_{e^{\prime}>e} \int_{\Gamma_{e^{\prime}}} \tau_{u}\left(\frac{\partial \mathbf{u}_{e}}{\partial n_{e}}+\frac{\partial \mathbf{u}_{e^{\prime}}}{\partial n_{e^{\prime}}}\right)^{2} d \Gamma\right)+\left(\frac{\beta}{1+\beta}\right)\left|p^{h}\right|_{\alpha_{e}, h_{e}, \mu, H^{1}\left(\Omega_{e}\right)}\right)^{\frac{1}{2}}, \\
& C_{3}=(3)^{\frac{1}{2}} \sup \left\{\left(\mu \frac{\beta}{1-\beta}\right)^{\frac{1}{2}} C^{*, F o r t i n}, C_{1}^{c} \widetilde{C}\left(\frac{1}{1-\beta}\right)^{\frac{1}{2}}, C_{2}\right\} .
\end{aligned}
$$


Combining the classic inequality (A.1) with $\gamma=1$ and (A.19) we find

$$
\begin{aligned}
& A^{h}\left(\mathbf{u}^{h}, p^{h}, \mathbf{v}^{h}, 0\right) \geqslant \frac{1}{2}\left(\sum_{e=1}^{n e}\left|\frac{\bar{p}_{e}^{h}}{\mu^{1 / 2}}\right|_{L^{2}\left(\Omega_{e}\right)}^{2}\right)-C_{4}\left(\sum _ { e = 1 } ^ { n e } ( 1 - \beta ) \left(\left|\mathbf{u}^{h}\right|_{\mu, H^{1}\left(\Omega_{e}\right)}^{2}+\left|\mathbf{u}^{h}\right|_{\mu, \ell, \text { Lap }}^{2}+\sum_{i=1}^{n} \int_{\Omega_{e}} \frac{\alpha_{e}\left(h_{e}\right)^{2}}{\mu}\left|R_{i}\left(\mathbf{u}^{h}, p^{h}\right)\right|^{2} d \Omega\right.\right. \\
& \left.\left.\quad+\sum_{e^{\prime}>e} \int_{\Gamma_{e e^{\prime}}} \tau_{u}\left(\frac{\partial \mathbf{u}_{e}}{\partial n_{e}}+\frac{\partial \mathbf{u}_{e^{\prime}}}{\partial n_{e^{\prime}}}\right)^{2} d \Gamma\right)+\left(\frac{\beta}{1+\beta}\right)\left|p^{h}\right|_{\alpha_{e}}, h_{e}, \mu, H^{1}\left(\Omega_{e}\right)^{2}\right), .
\end{aligned}
$$

where $C_{4}=\left(C_{3}\right)^{\frac{1}{2}} / 2$. It follows from the norm (45) that

$$
A^{h}\left(\mathbf{u}^{h}, p^{h}, \mathbf{v}^{h}, 0\right) \geqslant \frac{1}{2} \sum_{e=1}^{n e}\left(\left\|\frac{\bar{p}_{e}^{h}}{\mu^{1 / 2}}\right\|_{L^{2}\left(\Omega_{e}\right)}^{2}\right)-C_{4}\left(\left\|\left|\left(\mathbf{u}^{h}, p^{h}\right)\right|\right\|_{\beta, h, \Omega}^{2}-\sum_{e=1}^{n e}\left(\left\|\frac{\bar{p}_{e}^{h}}{\mu^{1 / 2}}\right\|_{L^{2}\left(\Omega_{e}\right)}^{2}\right)\right) .
$$

(3) Choosing

$$
\theta=\frac{1}{1+C_{4}+\frac{1}{4}}
$$

and setting

$$
\mathbf{w}^{h}=(1-\theta) \mathbf{u}^{h}+\theta \mathbf{v}^{h} \text { and } q^{h}=(1-\theta) p^{h},
$$

and by combining (A.7) with (A.22) $(k>1)$, we find

$$
A^{h}\left(\mathbf{u}^{h}, p^{h}, \mathbf{w}^{h}, q^{h}\right) \geqslant+\left((1-\theta)-\theta C_{4}\right)\left(\left\|\mid\left(\mathbf{u}^{h}, p^{h}\right)\right\|_{\beta, h, \Omega}^{2}-\left(\sum_{e=1}^{n e}\left\|\frac{\bar{p}_{e}^{h}}{\mu^{1 / 2}}\right\|_{L^{2}\left(\Omega_{e}\right)}^{2}\right)\right)+\frac{\theta}{2}\left(\sum_{e=1}^{n e}\left\|\frac{\bar{p}_{e}^{h}}{\mu^{1 / 2}}\right\|_{L^{2}\left(\Omega_{e}\right)}^{2}\right),
$$

and therefore it follows that

$$
A^{h}\left(\mathbf{u}^{h}, p^{h}, \mathbf{w}^{h}, q^{h}\right) \geqslant \frac{\theta}{4}\left\|\left|\left(\mathbf{u}^{h}, p^{h}\right)\right|\right\|_{\beta, h, \Omega}^{2} .
$$

Since

$$
\left(\mathbf{w}^{h}, p^{h}\right)=(1-\theta)\left(\mathbf{u}^{h}, p^{h}\right)+\theta\left(\mathbf{v}^{h}, 0\right),
$$

we have by triangular inequality, (45), (50) and (A.9) that

$$
\begin{aligned}
& \left\|\left|\left(\mathbf{w}^{h}, q^{h}\right)\right|\right\|_{\beta, h, \Omega} \leqslant(1-\theta)\left\|\left|\left(\mathbf{u}^{h}, p^{h}\right)\right|\right\|_{\beta, h, \Omega}+\theta\left\|\left|\left(\mathbf{v}^{h}, 0\right)\right|\right\|_{\beta, h, \Omega} \leqslant\left(\left(1-\beta^{2}\right) \mu\right)^{\frac{1}{2}} C_{5}\left\|\left|\left(\mathbf{u}^{h}, p^{h}\right)\right|\right\|_{\beta, h, \Omega}, \\
& C_{5}=C^{*, \text { Fortin }}\left(1+\lambda\left(\ell, M^{h}\right)\right) .
\end{aligned}
$$

Setting

$$
C_{\text {InfSup }}=\frac{\theta}{4\left(\left(1-\beta^{2}\right) \mu\right)^{\frac{1}{4}} C_{5}},
$$

finally yields

$$
A^{h}\left(\mathbf{u}^{h}, p^{h}, \mathbf{w}^{h}, q^{h}\right) \geqslant C_{\text {InfSup }}\left\|\left|\left(\mathbf{u}^{h}, p^{h}\right)\right|\right\|_{\beta, h, \Omega}\left\|\left|\left(\mathbf{w}^{h}, q^{h}\right)\right|\right\|_{\beta, h, \Omega},
$$

and the result follows immediately, completing the proof.

\section{References}

[1] G. Engel, K. Garikipati, T.J.R. Hughes, M.G. Larson, L. Mazzei, R.L. Taylor, Continuous/discontinouos finite element approximations of fourth-order elliptic problems in structural and continuum mechanics with applications to thin beams and plates, and strain gradient elasticity, Comput. Meth. Appl. Mech. Eng. 191 (2002) 3669-3750.

[2] S.C. Brenner, An a posteriori error estimator for a quadratic $C^{0}$-interior penalty method for biharmonic problem, J. Numer. Anal. 30 (2010) $777-798$.

[3] S.C. Brenner, L.-Y. Sung, C0 interior penalty methods for fourth order elliptic boundary value problems on polygonal domains, J. Sci. Comput. 22-23 (13) (2005) 83-118.

[4] S.-A. Papanicolopulos, A. Zervos, I. Vardoulakis, Discretization of Gradient Elasticity Problems Using C1 Finite Elements, in: G.A. Maugin, A.V. Metrikine (Eds.), Mechanics of Generalized Continua: One Hundred Years After the Cosserats, Springer, 2010, p. 269277.

[5] J.Jr. Douglas, T. Dupont, P. Percell, R. Scott, A family of $C^{1}$ finite elements with optimal approximations properties for various Galerkin for 2nd and 4th order problems, R.A.I.R.O. Model. Math. Anal. Numer. 13 (1979) 227-255.

[6] G.N. Wells, K. Garikipati, L. Molari, A discontinuous Galerkin formulation for a strain gradient-dependent continuum model, Comput. Methods Appl. Mech. Eng. 193 (33-35) (2004) 3633-3645.

[7] G.N. Wells, E. Kuhl, K. Garikipati, A discontinuous Galerkin method for the Cahn-Hilliard equation, J. Comput. Phys. 218 (2006) $860-877$.

[8] G.N. Wells, N.T. Dung, A C discontinuous Galerkin formulation for Kirchhoff plates, Comput. Methods Appl. Mech. Eng. 196 (2007) 3370-3380.

[9] F. Brezzi, M. Fortin, Mixed and hybrid finite element methods, Springer Series in Computational Mathematics, vol. 15 , Springer-Verlag, 1991. 
[10] J. L Guermond, A. Ern, A Theory and Pratictice of Finite Elements, Springer-Verlag, New York, 2004.

[11] J. Karam, A.F.D. Loula, A non-standard application of Babuska-Brezzi theory to finite element analysis of Stokes problem, Math. Appl. Comp. 10 (1991) 243-262.

[12] P.M. Gresho, R.L. Sani, Incompressible flow and the finite element method, Isothermal Laminar Flow, vol. 2, Wiley, England, 1998.

[13] F. Brezzi, R.S. Falk, stablility of higher-order Hood-Taylor methods, SIAM J. Numer. Anal. 28 (1991) 581-590.

[14] T. Barth, P. Bochev, J. Shadid, M. Gunzburger, A taxonomy of consistenly stabilized finite element methods for the Stokes problem, SIAM J. Sci. Comp. 25 (2004) 1585-1607.

[15] L.P. Franca, S.L. Frey, Stabilized finite element methods: II. The incompressible NavierStokes equations, Comput. Methods Appl. Mech. Eng. 99 (1992) 209-233.

[16] T.J.R. Hughes, L.P. Franca, A new finite element formulation for computational fluid dynamics: VII. The Stokes problem with various wellposed boundary conditions: symmetric formulations that converge for all velocity/pressure spaces, Comput. Methods Appl. Mech. Eng. 65 (1987) 85-96.

[17] T.J.R. Hughes, L.P. Franca, M.A. Balestra, A new finite element formulation for computational fluid dynamics: VIII. The Glerkin/least square methods for advective-diffusive equations, Comput. Methods Appl. Mech. Eng. 73 (1989) 173-189.

[18] L.P. Franca, T.J.R. Hughes, Convergence analyses of Galerkin least-squares methods for advective-diffusive forms of the Stokes and incompressible Navier-Stokes equations, Comput. Methods Appl. Mech. Eng. 105 (1993) 285-298.

[19] L.P. Franca, R. Stemberg, Error analysis of some Galerkin least squares methods for the elasticity equations, SIAM J. Numer. Anal. 28 (1991) $1680-1697$.

[20] T.-Y. Kim, J.D. Dolbow, E. Fried, A numerical method for a second-gradient theory of incompressible fluid flow, J. Comput. Phys. 223 (2007) 551-570.

[21] R.A. Adams, Sobolev Spaces, Academic Press, New York, 1975.

[22] E. Fried, M.E. Gurtin, Tranctions, balances, and boundary conditions for nonsimple materials with applications to liquid flow at small length scales, Arch. Ration. Mech. Anal. 182 (2006) 513-554.

[23] E. Fried, M.E. Gurtin, A continuum mechanical theory for turbulence: a generalized NavierStokes- $\alpha$ equation with boundary conditions, Theor. Comput. Fluid Dyn. 22 (2008) 433-470.

[24] J. Petera, J.F.T. Pittman, Isoparametric hermite elements, Int. J. Numer. Mech. Eng. 37 (1994) 3489-3519.

[25] T.-Y. Kim, J.E. Dolbow, An edge-bubble stabilized finite element method for fourth-order parabolic problems, Finite Elem. Anal. Des. 45 (2009) $485-$ 494.

[26] J. Blasco, An anisotropic pressure-stabilized finite element method for incompressible flow problems, Comput. Methods Appl. Mech. Eng. 197 (2008) $3712-3723$.

[27] K. Xia, H. Yao, A Galerkin/least-square finite element formulation for nearly incompressible elasticity/stokes flow, Appl. Math. Model. 31 (2007) 513529.

[28] E.G.D. Carmo, J.P.L. Santos, W.J. Mansur, Uma determinação sistematíca do parâmetro de calibração do método Galerkin Least Square (GLS), In 30 ${ }^{\text {th }}$ Congresso Ibero-Latino-Americano de Métodos Computacionais em Engenharia, Armação dos Búzios, Brazil (2009).

[29] J.P.L. Santos, Adaptive strategies for mixed finite element applied to viscoelasticity and incompressible linear models, D.Sc. thesis, Universidade Federal do Rio de Janeiro, Rio de Janeiro, 2011 (in Portuguese).

[30] B.J. Kirby, Micro- and Nanoscale Fluid Mechanics: Transport in Microfluidic Devices, Cambridge University Press, New York, 2010.

[31] M. Fortin, An analysis of the convergence of mixed finite element methods, RAIRO Anal. Numérique 11 (1977) 341-354.

[32] M. Fortin, Old and new finite elements for incompressible flows, Int. J. Numer. Methods Fluids 1 (1981) 347-364. 